\title{
VIBRATION OF AN AXIALLY LOADED HETEROGENEOUS PINNED-PINNED BEAM WITH AN INTERMEDIATE ROLLER SUPPORT
}

\author{
L. Kiss, G. Szeidl And M. AbDerrazeK \\ Institute of Applied Mechanics, University of Miskolc \\ 3515 Miskolc-Egyetemváros, Hungary \\ mechkiss@uni-miskolc.hu, gyorgy.szeidl@uni-miskolc.hu \\ abderrazekmessoudi1995@gmail.com
}

[Received: September 19, 2021; Accepted: November 3, 2021]

Dedicated to Professor Tibor Czibere on the occasion of his 90th birthday

\begin{abstract}
The present paper is devoted to the issue of what effect the axial load (compressive or tensile) has on the eigenfrequencies of a heterogeneous pinned-pinned beam with an intermediate roller support (called a PrsP beam). This problem is a three point boundary value problem (eigenvalue problem) associated with homogeneous boundary conditions. If the Green functions of the three point boundary value problem (BVP) are known the eigenvalue problem that provide the eigenfrequencies for the beam loaded axially can be transformed into an eigenvalue problem governed by a homogeneous Fredholm integral equation. The later eigenvalue problems can be reduced to an algebraic eigenvalue problem which then can be solved numerically by using an effective solution algorithm which is based on the boundary element method.
\end{abstract}

Mathematical Subject Classification: 34B27, 45c05, 74K10

Keywords: Heterogeneous beam, axially loaded, three point boundary value problem, vibration, Green's function, Fredholm integral equation

\section{INTRODUCTION}

Beams are quite often used as various structural elements because of their favorable load-bearing capabilities. Various such problems have been solved [1-3]. Nowadays, nonhomogeneous (heterogeneous or inhomogeneous) curved beams are also becoming more and more common. The benefits of such structural members can be the reduced weight and the higher strength. A class of inhomogeneity, namely, cross-sectional inhomogeneity means that the material parameters, like the Young modulus, are functions of the cross-sectional coordinates.

As for vibrations of beams, there are some recent results that must be mentioned. Bizzi et al. give an analytical solution to the mode-shape equation of Timoshenko beams vibrating under gravity loading in [4]. Ondra and Titurus 5 study systems of beams with tendon loading. The effect of the number and location of the attachment points is investigated on the vibrations of these systems is investigated. Bozyigit et al. 6 aim to find the effectiveness of the Adomian decomposition method and the differential transformation method on the vibrations of axially loaded Timoshenko beams. Mirzabeigy and Madoliat 7 focus on the vibrations of axially loaded beams resting on variable elastic foundation while $\mathrm{Wu}$ and Chang [8] tackle the vibrations of 
axially loaded multi-step Timoshenko beams carrying multiple concentrated elements. The continuous mass transfer matrix is used in the later article to find solutions.

At the same time, it seems that, meanwhile the Green function is commonly used for various straight beam problems. Lueschen et al 9] consider Timoshenko and EulerBernoulli beams - the Green function is given in closed-form for vibration problems. Foda and Abduljabbar [10] deal with the dynamic response of an Euler-Bernoulli beam finding the response of the beam to a moving mass. Kukla and Zamojska make some efforts in [11 to tackle the vibrations of an axially loaded tapered beam. The beam can consist of an arbitrary number of segments so the model can serve as approximation for continuously varying cross-sections. Failla and Santini [12] solve the bending problem of discontinuous beams - there are constraints along the beam axis and flexural stiffness jumps. An efficient algorithm is developed based on the Green function.

Based on the above literature research, in this article, it is our main objective to clarify what effect the axial force has on the eigenfrequencies of a pinned-pined beam with an intermediate roller support. The material is linearly elastic, isotropic. However, the elastic coefficients can change over the cross-section - this material behavior is called cross-sectional inhomogeneity. The governing equations of the corresponding equilibrium problem constitute a three-point boundary value problem. Its solution can be given in a closed form provided that the Green function is known. By utilizing the definition of the Green function we calculate the elements which make possible to calculate it. With the Green function the three-point eigenvalue problem that provides eigenfrequencies as a function of the axial force is replaced by a homogeneous Fredholm integral equation in which the Green function is the kernel. Then the eigenvalue problem governed by the homogeneous Fredholm integral equation is reduced to an algebraic eigenvalue problem by using the boundary element technique.

The paper is organized in five sections. Section 2 presents the differential equation of the problem and the corresponding boundary and continuity conditions in a dimensionless formulation. Section 3 provides the definition of the Green function for three-point boundary value problems. It is worth mentioning that the definition is a constructive one since it makes the calculation of the Green functions possible for the cases of compressive and tensile forces possible. The calculations are detailed in Section 4. The numerical results are presented in Section 5. The last section contains the conclusions.

\section{Differential equations}

2.1. Governing equations. The pinned-pinned beam with an intermediate roller support (PrsP beam) is shown in Figure 1. The axial force $N(N>0)$ is compressive in this Figure. The beam has a uniform cross section throughout its length. The $E$-weighted centerline [13] (called centerline for short) of the beam coincides with the axis $\hat{x}$ of the coordinate system $\hat{x}, \hat{y}, \hat{z}$. Its origin is at the left end of the centerline. It is assumed that the coordinate plane $\hat{x} \hat{z}$ is a symmetry plane of the beam. It is also assumed that the modulus of elasticity $E$ satisfies the relation $E(\hat{y}, \hat{z})=E(-\hat{y}, \hat{z})$ over 


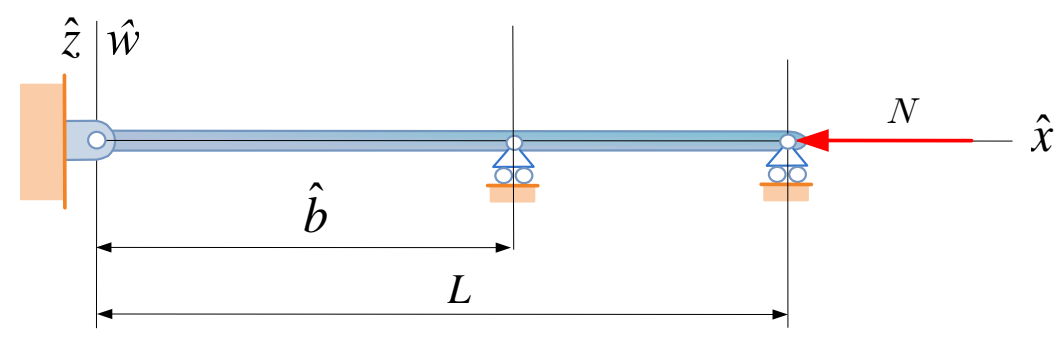

Figure 1.

the cross section $A$, which means that (a) it is an even function of $\hat{y}$ and (b) it is independent of the coordinate $\hat{x}$. In this case we speak about cross sectional heterogeneity.

We remark that the $E$-weighted first moment $Q_{\hat{y}}$ of the cross section is zero in this coordinate system $[13$ :

$$
Q_{\hat{y}}=\int_{A} \hat{y} E(\hat{y}, \hat{z}) \mathrm{d} A=0
$$

The length of the beam is $L$, the position of the middle support is given by $\hat{b}$.

Equilibrium problems of Euler-Bernoulli beams with cross sectional heterogeneity are governed by the ordinary differential equation:

$$
\frac{\mathrm{d}^{4} \hat{w}}{\mathrm{~d} \hat{x}^{4}} \pm \hat{\mathcal{N}} \frac{\mathrm{d}^{2} \hat{w}}{\mathrm{~d} \hat{x}^{2}}=\frac{\hat{f}_{z}}{I_{e y}}, \quad \hat{\mathcal{N}}=\frac{N}{I_{e y}}
$$

where the sign of $\hat{\mathcal{N}}$ is (positive)[negative] if the axial force is (comressive)[tensile], $\hat{w}(x)$ is the vertical displacement of the material points on the centerline, $\hat{f}_{z}(\hat{x})$ is the intensity of the distributed load acting on the centerline and $I_{e y}$ is defined by the equation

$$
I_{e y}=\int_{A} E(\hat{y}, \hat{z}) z^{2} \mathrm{~d} A,
$$

where $A$ is the area of the cross section. If $E$ is constant the beam is homogeneous and

$$
I_{e y}=I E, \quad I=\int_{A} z^{2} \mathrm{~d} A
$$

in which $I$ is the moment of inertia. It is noted that the effect of the material composition on $I_{e y}$ is demonstrated through examples in 14].

In what follows we shall use dimensionless variables defined by the following relations

$$
\begin{aligned}
& x=\hat{x} / L, \quad \xi=\hat{\xi} / L, \quad w=\hat{w} / L, \\
& y=\frac{\mathrm{d} \hat{w}}{\mathrm{~d} \hat{x}}=\frac{\mathrm{d} w}{\mathrm{~d} x}, \quad b=\hat{b} / \ell, \quad \ell=\left.\frac{\hat{x}}{L}\right|_{\hat{x}=L}=1,
\end{aligned}
$$


where $\hat{\xi}$ is also a coordinate on the axis $x$ introduced here for our later considerations. Applying dimensionless quantities to equation (2.1) we have

$$
\frac{\mathrm{d}^{4} w}{\mathrm{~d} x^{4}} \pm \mathcal{N} \frac{\mathrm{d}^{2} w}{\mathrm{~d} x^{2}}=f_{z}, \quad \mathcal{N}=L^{2} \hat{\mathcal{N}}=\frac{L^{2} N}{I_{e y}} \quad f_{z}=\frac{L^{3} \hat{f}_{z}}{I_{e y}}
$$

Table 1.

\begin{tabular}{|c|c|}
\hline \multicolumn{2}{|c|}{ Boundary conditions } \\
\hline$w(0)=0, w^{(2)}(0)=0$ & $w(\ell)=0, w^{(2)}(\ell)=0$ \\
\hline \hline \multicolumn{2}{|c|}{ Continuity conditions } \\
\hline \multirow{2}{|c|}{$w(b+0)=0$} \\
$w^{(1)}(b-0)=w^{(1)}(b+0)$ \\
$w^{(2)}(b-0)=w^{(2)}(b+0)$ \\
\hline
\end{tabular}

Equation 2.5 is associated with the boundary and continuity conditions presented in Table 1.

We remark that the general solution of the homogeneous differential equation

$$
w^{(4)} \pm \mathcal{N} w^{(2)}=0, \quad w^{(n)}= \begin{cases}w & \text { if } n=0 \\ \frac{\mathrm{d}^{n} w}{\mathrm{~d} x^{n}} & \text { if } n=1,2, \ldots\end{cases}
$$

is very simple, i.e.,

$$
w=\sum_{\ell=1}^{4} a_{\ell} w_{\ell}(x)=a_{1}+a_{2} x+a_{3} \cos p x+a_{4} \sin p x, \quad p=\sqrt{\mathcal{N}}
$$

if the sign of $\mathcal{N}$ is positive $-a_{1}, \ldots, a_{4}$ are undetermined integration constants - and

$$
w=\sum_{\ell=1}^{4} a_{\ell} w_{\ell}(x)=a_{1}+a_{2} x+a_{3} \cosh p x+a_{4} \sinh p x, \quad p=\sqrt{\mathcal{N}} .
$$

if the sign of $\mathcal{N}$ is negative. Note that $a_{1}, \ldots, a_{4}$ are different integration constants in equations (2.6b) and 2.6c).

If we know the Green function $G(x, \xi)$ for the boundary value problem determined by ODE 2.5 and the boundary and continuity conditions presented in Table 1 the solution for the dimensionless deflection $w(x)$ due to the dimensionless load $f_{z}(\xi)$ is given by the following integral:

$$
w(x)=\int_{0}^{\ell} G(x, \xi) f_{z}(\xi) \mathrm{d} \xi
$$

The definition of the Green function we need is presented in Section 3 


\section{Green FunCtion For three-Point boundary VAlue PROBlems}

3.1. Definition. We shall consider the in-homogeneous ordinary differential equation

$$
L[y(x)]=r(x),
$$

where the differential operator of order $2 k$ is defined by the following equation

$$
L[y(x)]=\sum_{n=0}^{2 k} p_{n}(x) y^{(n)}(x)
$$

in which $k$ is a natural number, the functions $p_{n}(x)$ and $r(x)$ are continuous and $p_{2 k}(x) \neq 0$ if $x \in[0, \ell](\ell>0)$. Moreover let $b$ be an inner point in the interval $[0, \ell]$ : $b=\ell_{1}, \ell-b=\ell_{2}$ and $\ell_{1}+\ell_{2}=\ell$.

We assume that the inhomogeneous differential equation 3.1 is associated with homogeneous boundary and continuity conditions given by the following equations:

$$
\begin{array}{rlr}
U_{0 r}[y] & =\sum_{n=0}^{2 k} \alpha_{n r I} y_{I}^{(n-1)}(0)=0, & r=1,2, \ldots, k \\
U_{b r}[y]=\sum_{n=0}^{2 k} \beta_{n r I} y_{I}^{(n-1)}(b)-\sum_{n=0}^{2 k} \beta_{n r I I} y_{I I}^{(n-1)}(b)=0, & r=1,2, \ldots, 2 k \\
U_{\ell r}[y]=\sum_{n=0}^{2 k} \gamma_{n r I I} y_{I I}^{(n-1)}(\ell)=0, & r=1,2, \ldots, k
\end{array}
$$

The Latin subscripts $I$ and $I I$ refer successively to the intervals $[0, b]$ and $[b, \ell]: y_{I}$ and $y_{I I}$ are the solutions to the differential equation in the interval $I$ and $I I$ while $\alpha_{n r I}, \beta_{n r I}, \beta_{n r I I}$ and $\gamma_{n r I I}$ are arbitrary constants.

In accordance with equation $(2.7)$ solution of the three-point boundary value problem (3.1), (3.2) and (3.3) is sought in the following form:

$$
y(x)=\int_{0}^{\ell} G(x, \xi) r(\xi) \mathrm{d} \xi,
$$

where the function $G(x, \xi)$ is the Green function defined by the following formulas and properties $[15$ :

Formulas:

$$
G(x, \xi)= \begin{cases}G_{1 I}(x, \xi) & \text { if } x, \xi \in[0, b] \\ G_{2 I}(x, \xi) & \text { if } x \in[b, \ell] \text { and } \xi \in[0, b] \\ G_{1 I I}(x, \xi) & \text { if } x \in[0, b] \text { and } \xi \in[b, \ell] \\ G_{2 I I}(x, \xi) & \text { if } x, \xi \in[b, \ell]\end{cases}
$$

Properties:

1. The function $G_{1 I}(x, \xi)$ is a continuous function of $x$ and $\xi$ if $0 \leq x \leq \xi \leq b$ and $0 \leq \xi \leq x \leq b$. In addition it is $2 k$ times differentiable with respect to $x$ and the derivatives

$$
\frac{\partial^{n} G_{1 I}(x, \xi)}{\partial x^{n}}=G_{1 I}(x, \xi)^{(n)}(x, \xi), \quad n=1,2, \ldots, 2 k
$$


are also continuous functions of $x$ and $\xi$ in the triangles $0 \leq x \leq \xi \leq b$ and $0 \leq \xi \leq$ $x \leq b$.

2. Let $\xi$ be fixed in $[0, b]$. The function $G_{1 I}(x, \xi)$ and its derivatives

$$
G_{1 I}^{(n)}(x, \xi)=\frac{\partial^{n} G_{1 I}(x, \xi)}{\partial x^{n}}, \quad n=1,2, \ldots, 2 k-2
$$

should be continuous for $x=\xi$ :

$$
\begin{aligned}
\lim _{\varepsilon \rightarrow 0}\left[G_{1 I}^{(n)}(\xi+\varepsilon, \xi)-G_{1 I}^{(n)}(\xi-\varepsilon, \xi)\right]= \\
\quad=\left[G_{1 I}^{(n)}(\xi+0, \xi)-G_{1 I}^{(n)}(\xi-0, \xi)\right]=0 \quad n=0,1,2, \ldots 2 k-2
\end{aligned}
$$

The derivative $G_{1 I}^{(2 k-1)}(x, \xi)$ should, however, have a jump if $x=\xi$ :

$$
\begin{aligned}
\lim _{\varepsilon \rightarrow 0}\left[G_{1 I}^{(2 k-1)}(\xi+\varepsilon, \xi)-G_{1 I}^{(2 k-1)}(\xi-\varepsilon, \xi)\right]= \\
=\left[G_{1 I}^{(2 k-1)}(\xi+0, \xi)-G_{1 I}^{(2 k-1)}(\xi-0, \xi)\right]=\frac{1}{p_{2 k}(\xi)} .
\end{aligned}
$$

In contrast to this, $G_{2 I}(x, \xi)$ and its derivatives

$$
G_{2 I}^{(n)}(x, \xi)=\frac{\partial^{n} G_{2 I}(x, \xi)}{\partial x^{n}}, \quad n=1,2, \ldots, 2 k
$$

are all continuous functions for any $x$ in $[b, \ell]$.

3. Let $\xi$ be fixed in $[b, \ell]$. The function $G_{1 I I}(x, \xi)$ and its derivatives

$$
G_{1 I I}^{(n)}(x, \xi)=\frac{\partial^{n} G_{1 I I}(x, \xi)}{\partial x^{n}}, \quad n=1,2, \ldots, 2 k
$$

are all continuous functions for any $x$ in $[0, b]$.

4. Though the function $G_{2 I I}(x, \xi)$ and its derivatives

$$
G_{2 I I}^{(n)}(x, \xi)=\frac{\partial^{n} G_{2 I I}(x, \xi)}{\partial x^{n}}, \quad n=1,2, \ldots, 2 k-2
$$

are also continuous for $x=\xi$ :

$$
\begin{aligned}
& \lim _{\varepsilon \rightarrow 0} {\left[G_{2 I I}^{(n)}(\xi+\varepsilon, \xi)-G_{2 I I}^{(n)}(\xi-\varepsilon, \xi)\right]=} \\
& \quad=\left[G_{2 I I}^{(n)}(\xi+0, \xi)-G_{2 I I}^{(n)}(\xi-0, \xi)\right]=0 \quad n=0,1,2, \ldots 2 k-2
\end{aligned}
$$

the derivative $G_{2 I I}^{(2 k-1)}(x, \xi)$ should, however, have a jump if $x=\xi$ :

$$
\begin{aligned}
\lim _{\varepsilon \rightarrow 0}\left[G_{2 I I}^{(2 k-1)}\right. & \left.(\xi+\varepsilon, \xi)-G_{21 I}^{(2 k-1)}(\xi-\varepsilon, \xi)\right]= \\
& =\left[G_{2 I I}^{(2 k-1)}(\xi+0, \xi)-G_{2 I I}^{(2 k-1)}(\xi-0, \xi)\right]=\frac{1}{p_{2 k}(\xi)}
\end{aligned}
$$


5. Let $\alpha$ be an arbitrary but finite non-zero constant. For a fixed $\xi \in[0, \ell]$ the product $G(x, \xi) \alpha$ as a function of $x(x \neq \xi)$ should satisfy the homogeneous differential equation

$$
M[G(x, \xi) \alpha]=0 .
$$

6. The product $G(x, \xi) \alpha$ as a function of $x$ should satisfy both the boundary conditions and the continuity conditions

$$
\begin{array}{r}
U_{0 r}[G]=\sum_{n=1}^{2 k} \alpha_{n r I} G^{(n-1)}(0)=0, \quad r=1, \ldots, k \\
U_{b r}[G]=\sum_{n=1}^{2 k}\left(\beta_{n r I} G^{(n-1)}(b-0)-\beta_{n r I I} G^{(n-1)}(b+0)\right)=0, \quad r=1, \ldots, 2 k \\
U_{\ell r}[G]=\sum_{n=1}^{2 k} \gamma_{n r I I} G^{(n-1)}(\ell)=0, \quad r=1, \ldots, k
\end{array}
$$

The above continuity conditions should be satisfied by the function pairs $G_{1 I}(x, \xi)$, $G_{2 I}(x, \xi)$ and $G_{1 I I}(x, \xi), G_{2 I I}(x, \xi)$ as well.

REMARK 1. It can be proved by utilizing the previous definition that integral (3.4) satisfies differential equation and boundary conditions (3.3) [15].

REMARK 2. If the boundary value problem defined by 3.1 and $(3.3)$ is self adjoint then the Green function is symmetric 15:

$$
G(x, \xi)=G(\xi, x)
$$

It can be checked with ease utilizing the corresponding definition presented in 15 that the three-point boundary value problem defined by differential equation $2.6 \mathrm{a}$ and the boundary and continuity condition given in Table 1 is self-adjoint.

\subsection{Calculation of the Green functions.}

3.2.1. Introductory remarks. Let us denote the linearly independent particular solutions of the homogeneous ordinary differential equation

$$
L[y(x)]=0
$$

by

$$
y_{1}(x), y_{2}(x), y_{3}(x), \ldots, y_{2 k}(x) .
$$

Since the general solution is a linear combination of the particular solutions it can be given in the following form:

$$
y(x)=\sum_{n=1}^{2 k} \mathcal{A}_{n} y_{n}(x)
$$

where the coefficients $\mathcal{A}_{n}$ are arbitrary integration constants. The Green function should satisfy the homogeneous differential equation (3.15). Therefore it follows that it can be given as a linear combination of the particular solutions $y_{n}(x)$, i.e., by equation (3.17). It is a further problem how to find the coefficients $\mathcal{A}_{n}$. Subsections 3.3 and 3.4 deal with this issue. 
3.3. Calculation of the Green function if $\xi \in[0, b]$. It is obvious that the integration constants $\mathcal{A}_{n}$ should be different in the two triangular domains $0 \leq x \leq \xi \leq b$

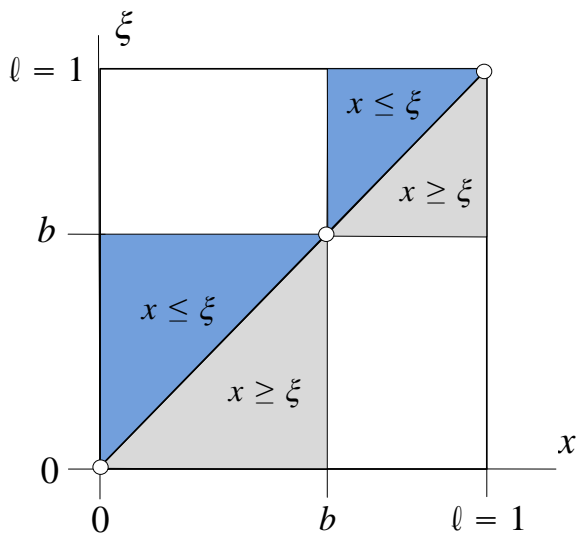

Figure 2.

and $0 \leq \xi \leq x \leq b$. For this reason we shall apply the following assumptions:

(a)

$$
\begin{array}{ll}
G_{1 I}(x, \xi)=\sum_{\ell=1}^{2 k}\left(a_{\ell I}(\xi)+b_{\ell I}(\xi)\right) y_{\ell}(x), & x \leq \xi ; \\
G_{1 I}(x, \xi)=\sum_{\ell=1}^{2 k}\left(a_{\ell I}(\xi)-b_{\ell I}(\xi)\right) y_{\ell}(x), & x \geq \xi
\end{array}
$$

and

(b)

$$
G_{2 I}(x, \xi)=\sum_{\ell=1}^{2 k} c_{\ell I}(\xi) y_{\ell}(x), \quad x \in[b, \ell]
$$

where the coefficients $a_{\ell I}(\xi), b_{\ell I}(\xi)$ and $c_{\ell I}(\xi)$ are, in fact, unknown functions.

Note that the above representation for $G_{1 I}(x, \xi)$ and $G_{2 I}(x, \xi)$ ensures the fulfillment of Property 5 of the definition.

Continuity conditions $3.8 \mathrm{a}$ yield the following equations

$$
\sum_{\ell=1}^{2 k} b_{\ell I}(\xi) y_{\ell}^{(n)}(\xi)=0, \quad n=0,1,2, \ldots 2 k-2
$$

As regards the discontinuity condition $(3.8 \mathrm{~b})$ we get

$$
\frac{1}{p_{2 k}(\xi)}=\left[G^{(2 k-1)}(\xi+0, \xi)-G^{(2 k-1)}(\xi-0, \xi)\right]=-2 \sum_{\ell=1}^{k} b_{\ell I}(\xi) y_{\ell}^{(2 k-1)}(\xi) \text {. }
$$


Hence

$$
\sum_{\ell=1}^{2 k} b_{\ell I}(\xi) y_{\ell}^{(2 k-1)}(\xi)=-\frac{1}{2 p_{2 k}(\xi)} .
$$

Fulfillment of equations $(3.20 \mathrm{a}$ and $(3.20 \mathrm{~b}$ ensures the fulfillment of Properties 1,2 and 4 of the definition.

Continuity and discontinuity conditions 3.8 result in the inhomogeneous linear system of equations 3.20 for the unknowns $b_{\ell}(\xi)(\ell=1,2, \ldots, 2 k)$. Its determinant assumes the following form:

$$
\left|\begin{array}{cccc}
y_{1}(\xi) & y_{2}(\xi) & \ldots & y_{k}(\xi) \\
y_{1}^{(1)}(\xi) & y_{2}^{(1)}(\xi) & \ldots & y_{k}^{(1)}(\xi) \\
\ldots \ldots \ldots \ldots \ldots \ldots \ldots \ldots \ldots \ldots \ldots \ldots \ldots \ldots \\
y_{1}^{(2 k-1)}(\xi) & y_{2}^{(2 k-1)}(\xi) & \ldots & y_{2 k}^{(2 k-1)}(\xi)
\end{array}\right| .
$$

This determinant is the Wronskian 11 of differential equation 3.15 which is not zero since the particular solutions $y_{\ell}(x)$ are linearly independent. This means that we can always find a unique solution for the coefficients $b_{\ell I}(\xi)$ in representation (3.18) of the Green function.

Utilizing the boundary and continuity conditions 3.13 the following equations can be obtained for the unknown coefficients $a_{\ell I}(\xi)$ and $c_{\ell I}(\xi)$ :

$$
\begin{gathered}
U_{0 r}[G]=\sum_{n=1}^{2 k} \alpha_{n r I} \sum_{\ell=1}^{2 k}\left(a_{\ell I}(\xi)+b_{\ell I}(\xi)\right) z_{\ell}(0)^{(n-1)}=0 \quad r=1,2, \ldots, k \\
U_{b r}[G]=\sum_{n=1}^{2 k}\left(\beta_{n r I} \sum_{\ell=1}^{2 k}\left(a_{\ell I}(\xi)-b_{\ell I}(\xi)\right) z_{\ell}(b)^{(n-1)}-\right. \\
\left.-\beta_{n r I I} \sum_{\ell=1}^{2 k} c_{\ell I}(\xi) z_{\ell}(b)^{(n-1)}\right)=0, \quad r=1,2, \ldots, 2 k \\
U_{c r}[G]=\sum_{n=1}^{2 k} \gamma_{n r I I} \sum_{\ell=1}^{2 k} c_{\ell I}(\xi) z_{\ell}(c)^{(n-1)}=0, \quad r=1,2, \ldots, k
\end{gathered}
$$

or

$$
\begin{array}{rlrl}
\sum_{\ell=1}^{2 k} a_{\ell I} U_{a r}\left[z_{\ell}\right] & =-\sum_{\ell=1}^{2 k} b_{\ell I} U_{a r}\left[z_{\ell}\right] & r=1,2, \ldots, k \\
\sum_{\ell=1}^{2 k} a_{\ell I} U_{b r I}\left[z_{\ell}\right]-\sum_{\ell=1}^{2 k} c_{\ell I} U_{b r I I}\left[z_{\ell}\right] & =\sum_{\ell=1}^{2 k} b_{\ell I} U_{b r I}\left[z_{\ell}\right] & & r=1,2, \ldots, 2 k \\
\sum_{\ell=1}^{2 k} c_{\ell I} U_{c r}\left[z_{\ell}\right] & =0 & r=1,2, \ldots, k
\end{array}
$$

\footnotetext{
${ }^{1}$ Józef Hoene-Wroński (1776-1853)
} 
Solutions for $a_{\ell I}(\xi)$ and $c_{\ell I}(\xi)$ exist if and only if the determinant of the linear equation system (3.23) is different from zero. The linear independence of the boundary and continuity conditions assures in general that the equation system 3.23 is solvable.

Fulfillment of equations 3.23 ensures the fulfillment of Property 6 of the definition.

3.4. Calculation of the Green function if $\xi \in[b, \ell=1]$. Now we shall assume that:

(a)

$$
\begin{array}{lll}
G_{1 I}(x, \xi)=\sum_{\ell=1}^{2 k}\left(a_{\ell I I}(\xi)+b_{\ell I I}(\xi)\right) y_{\ell}(x), & x \leq \xi ; & \\
G_{1 I}(x, \xi)=\sum_{\ell=1}^{2 k}\left(a_{\ell I I}(\xi)-b_{\ell I I}(\xi)\right) y_{\ell}(x), & x \geq \xi &
\end{array}
$$

and

(b)

$$
G_{2 I}(x, \xi)=\sum_{\ell=1}^{2 k} c_{\ell I}(\xi) y_{\ell}(x), \quad x \in[0, b]
$$

where the coefficients $a_{\ell I I}(\xi), b_{\ell I I}(\xi)$ and $c_{\ell I I}(\xi)$ are again unknown functions.

Note that the above representation for $G_{1 I I}(x, \xi)$ and $G_{2 I I}(x, \xi)$ ensures the fulfillment of Property 5 of the definition.

Continuity conditions 3.12a lead to the following equations:

$$
\sum_{\ell=1}^{2 k} b_{\ell I I}(\xi) y_{\ell}^{(n)}(\xi)=0, \quad n=0,1,2, \ldots 2 k-2
$$

As regards discontinuity condition $3.12 \mathrm{~b}$ we obtain

$$
\frac{1}{p_{2 k}(\xi)}=\left[G^{(2 k-1)}(\xi+0, \xi)-G^{(2 k-1)}(\xi-0, \xi)\right]=-2 \sum_{\ell=1}^{k} b_{\ell I I}(\xi) y_{\ell}^{(2 k-1)}(\xi) .
$$

Hence

$$
\sum_{\ell=1}^{2 k} b_{\ell I I}(\xi) y_{\ell}^{(2 k-1)}(\xi)=-\frac{1}{2 p_{2 k}(\xi)} .
$$

Continuity and discontinuity conditions $(3.12)$ yield the inhomogeneous linear system of equations $(3.26)$ for the unknowns $b_{\ell I I}(\xi)(\ell=1,2, \ldots, 2 k)$. Since these equation are formally the same as equations $(3.20)$ it follows that their determinant is again the Wronskian (3.21) and this fact guarantees their solvability. It also follows that $b_{\ell I I}=b_{\ell I}$. 
Note that fulfillment of equations $3.26 \mathrm{a}$ and $3.26 \mathrm{~b}$ ensures the fulfillment of Properties 1, 3 and 4 of the definition.

Utilizing now the boundary and continuity conditions 3.13 leads to the following linear equations for the unknown coefficients $a_{\ell I I}(\xi)$ and $c_{\ell I I}(\xi)$ :

$$
\begin{gathered}
U_{0 r}[G]=\sum_{n=1}^{2 k} \alpha_{n r I} \sum_{\ell=1}^{2 k} c_{\ell I I}(\xi) z_{\ell}(a)^{(n-1)}=0 \quad r=1,2, \ldots, k \\
U_{b r}[G]=\sum_{n=1}^{2 k}\left(\beta_{n r I} \sum_{\ell=1}^{2 k} c_{\ell I I}(\xi) z_{\ell}(b)^{(n-1)}-\right. \\
\left.-\beta_{n r I I} \sum_{\ell=1}^{2 k}\left(a_{\ell I I}(\xi)+b_{\ell I I}(\xi)\right) z_{\ell}(b)^{(n-1)}\right)=0, r=1,2, \ldots, 2 k \\
U_{\ell r}[G]=\sum_{n=1}^{2 k} \gamma_{n r I I} \sum_{\ell=1}^{2 k}\left(a_{\ell I I}(\xi)-b_{\ell I I}(\xi)\right) z_{\ell}(c)^{(n-1)}=0, \quad r=1,2, \ldots, k
\end{gathered}
$$

or

$$
\begin{array}{cc}
\sum_{\ell=1}^{2 k} a_{\ell I I} U_{0 r}\left[z_{\ell}\right]=0 & r=1,2, \ldots, k \\
\sum_{\ell=1}^{2 k} a_{\ell I I} U_{b r I}\left[z_{\ell}\right]-\sum_{\ell=1}^{2 k} b_{\ell I I} U_{b r I I}\left[z_{\ell}\right]=\sum_{\ell=1}^{2 k} c_{\ell I I} U_{b r I I}\left[z_{\ell}\right] & r=1,2, \ldots, 2 k \\
\sum_{\ell=1}^{2 k} b_{\ell I I} U_{\ell r}\left[z_{\ell}\right]=\sum_{\ell=1}^{2 k} c_{\ell I I} U_{\ell r}\left[z_{\ell}\right] & r=1,2, \ldots, k
\end{array}
$$

The linear equation system (3.27) is solvable for $a_{\ell I I}(\xi)$ and $b_{\ell I I}(\xi)$ if its determinant is different from zero. Since the boundary and continuity conditions are linearly independent equation system (3.27) is, in general, solvable.

If the determinants of the equation systems $(3.23)$ and 3.28 are different from zero then there Green function exits which satisfies the properties of the definition given in Section 3.1 .

Fulfillment of equations 3.28 ensures the fulfillment of Property 6 of the definition.

\section{Calculation of the Green function for PrsP beams}

4.1. PrsP beams subjected to a compressive force. If the axial force is compression it follows from equation (2.5) (or 2.6a) that equilibrium problems of PrsP beams are governed by the differential equation

$$
L[w(x)]=w^{(4)}+\mathcal{N} w^{(2)}=f_{z}
$$

associated with the boundary and continuity conditions in Table 1 . 
4.1.1. Green function if $\xi \in[0, b]$. In the sequel we shall follow the line of thought presented in Subsection 3.3. Recalling that the general solution of the homogeneous differential equation $L[w(x)]=0$ is given by $2.6 \mathrm{~b}$ the continuity and discontinuity conditions (3.20) result in the following equation system for $b_{\ell I}$ :

$$
\left[\begin{array}{cccc}
1 & \xi & \cos p \xi & \sin p \xi \\
0 & 1 & -p \sin p \xi & p \cos p \xi \\
0 & 0 & -p^{2} \cos p \xi & -p^{2} \sin p \xi \\
0 & 0 & p^{3} \sin p \xi & -p^{3} \cos p \xi
\end{array}\right]\left[\begin{array}{l}
b_{1} \\
b_{2} \\
b_{3} \\
b_{4}
\end{array}\right]=\left[\begin{array}{c}
0 \\
0 \\
0 \\
-\frac{1}{2}
\end{array}\right]
$$

REMARK 3. It has been taken into account here that $w(x)$ corresponds to $y(x), k=2$ and $p_{2 k}=1$.

Solution of this equation system assumes the following form:

$$
\left[\begin{array}{l}
b_{1 I} \\
b_{2 I} \\
b_{3 I} \\
b_{4 I}
\end{array}\right]=\frac{1}{2 p^{3}}\left[\begin{array}{c}
p \xi \\
-p \\
-\sin p \xi \\
\cos p \xi
\end{array}\right] \text {. }
$$

We proceed with the boundary and continuity conditions $(3.23)$. Recalling the boundary and continuity conditions in Table 1 and Remark 3. we obtain the following equation system:

(a) Boundary conditions at $x=0$ :

$$
\begin{aligned}
& a_{1 I} w_{1}(0)+a_{2 I} w_{2}(0)+a_{3 I} w_{3}(0)+a_{4 I} w_{4}(0)= \\
& =-b_{1 I} w_{1}(0)-b_{2 I} w_{2}(0)-b_{3 I} w_{3}(0)-b_{4 I} w_{4}(0), \\
& \begin{array}{r}
a_{1 I} w_{1}^{(2)}(0)+a_{2 I} w_{2 I}^{(2)}(0)+a_{3 I} w_{3}^{(2)}(0)+a_{4 I} w_{4}^{(2)}(0)= \\
=-b_{1 I} w_{1}^{(2)}(0)-b_{2 I} w_{2}^{(2)}(0)-b_{3 I} w_{3}^{(2)}(0)-b_{4 I} w_{4}^{(2)}(0) .
\end{array}
\end{aligned}
$$

(b) Continuity conditions at $x=b$ :

$$
\begin{gathered}
a_{1 I} w_{1}(b)+a_{2 I} w_{2}(b)+a_{3 I} w_{3}(b)+a_{4 I} w_{4}(b)= \\
=b_{1 I} w_{1}(b)+b_{2 I} w_{2}(b)+b_{3 I} w_{3}(b)+b_{4 I} w_{4}(b), \\
c_{1 I} w_{1}(b)+c_{2 I} w_{2}(b)+c_{3 I} w_{3}(b)+c_{4 I} w_{4}(b)=0, \\
a_{1 I} w_{1}^{(1)}(b)+a_{2 I} w_{2 I}^{(1)}(b)+a_{3 I} w_{3}^{(1)}(b)+a_{4 I} w_{4}^{(1)}(b)- \\
-c_{1 I} w_{1}^{(1)}(b)-c_{2 I} w_{2}^{(1)}(b)-c_{3 I} w_{3}^{(1)}(b)-c_{4 I} w_{4}^{(1)}(b)= \\
=b_{1 I} w_{1}^{(1)}(b)+b_{2 I} w_{2}^{(1)}(b)+b_{3 I} w_{3}^{(1)}(b)+b_{4 I} w_{4}^{(1)}(b), \\
a_{1 I} w_{1}^{(2)}(b)+a_{2 I} w_{2 I}^{(2)}(b)+a_{3 I} w_{3}^{(2)}(b)+a_{4 I} w_{4}^{(2)}(b)- \\
-c_{1 I} w_{1}^{(2)}(b)-c_{2 I} w_{2}^{(2)}(b)-c_{3 I} w_{3}^{(2)}(b)-c_{4 I} w_{4}^{(2)}(b)= \\
=b_{1 I} w_{1}^{(2)}(b)+b_{2 I} w_{2}^{(2)}(b)+b_{3 I} w_{3}^{(2)}(b)+b_{4 I} w_{4}^{(2)}(b) .
\end{gathered}
$$


(c) Boundary conditions at $x=\ell$ :

$$
\begin{gathered}
c_{1 I} w_{1}(\ell)+c_{2 I} w_{2}(\ell)+c_{3 I} w_{3}(\ell)+c_{4 I} w_{4}(\ell)=0, \\
c_{1 I} w_{1}^{(2)}(\ell)+c_{2 I} w_{2}^{(2)}(\ell)+c_{3 I} w_{3}^{(2)}(\ell)+c_{4 I} w_{4}^{(2)}(\ell)=0 .
\end{gathered}
$$

Using 2.6b boundary conditions (4.4) at $x=0$ yield

$$
a_{1 I}+a_{3 I}=-b_{1 I}-b_{3 I}, \quad a_{3 I}=-b_{3 I} .
$$

Hence

$$
\begin{aligned}
& a_{1 I}=-b_{1 I}=-\frac{1}{2 p^{2}} \xi, \\
& a_{3 I}=\frac{\sin p \xi}{2 p^{3}} .
\end{aligned}
$$

As regards continuity conditions 4.5 at $x=b$ and boundary conditions 4.6 at $x=\ell$ substituting (2.6b) yields

$$
\begin{gathered}
{\left[\begin{array}{cccccc}
b & \sin p b & 0 & 0 & 0 & 0 \\
0 & 0 & 1 & b & \cos p b & \sin p b \\
1 & p \cos p b & 0 & -1 & p \sin p b & -p \cos p b \\
0 & -\sin p b & 0 & 0 & \cos p b & \sin p b \\
0 & 0 & 1 & \ell & \cos p \ell & \sin p \ell \\
0 & 0 & 0 & 0 & \cos p \ell & \sin p \ell
\end{array}\right]\left[\begin{array}{c}
a_{2 I} \\
a_{4 I} \\
c_{1 I} \\
c_{2 I} \\
c_{3 I} \\
c_{4 i}
\end{array}\right]=} \\
=\frac{1}{2 p^{3}}\left[\begin{array}{c}
2 p \xi-p b-\sin p \xi \cos p b-\sin p(\xi-b) \\
0 \\
-p+2 p \sin p \xi \sin p b+p \cos p \xi \cos p b \\
2 \sin p \xi \cos p b-\cos p \xi \sin p b \\
0 \\
0
\end{array}\right]
\end{gathered}
$$

from where

$$
\begin{aligned}
& a_{2 I}= \\
& \begin{aligned}
= & \frac{1}{2 p^{3} \mathcal{D}_{c}}[(p(\ell-b) \sin p \ell-\sin b p \sin p(\ell-b))(2 p \xi-b p-\sin p(\xi-b)-\sin p \xi \cos b p)+ \\
& \quad-(\ell-b)(\sin b p \sin p(\ell-b))(-p+2 p \sin p \xi \sin b p+p \cos p \xi \cos b p)+ \\
& +(p(\ell-b) \sin b p \cos (p \ell-b p)-\sin b p \sin (p \ell-b p))(2 \sin p \xi \cos b p-\cos p \xi \sin b p)],
\end{aligned}
\end{aligned}
$$

$$
\begin{aligned}
& a_{4 I}= \\
& \begin{array}{r}
=\frac{1}{2 p^{3} \mathcal{D}_{c}}[-(\cos p \xi \sin p b-2 \sin p \xi \cos p b)(b \sin p(\ell-b)-p b(\ell-b) \cos p(\ell-b))- \\
\quad+(\ell-b)(\sin p(\ell-b))(\sin p(\xi-b)-2 p \xi+p b+\sin p \xi \cos p b)- \\
\quad-b(\ell-b) \sin p(\ell-b)(p-2 p \sin p \xi \sin p b-p \cos p \xi \cos p b)],
\end{array} \\
& \quad
\end{aligned}
$$




$$
\begin{aligned}
& c_{1 I}=\frac{1}{2 p^{3} \mathcal{D}_{c}}[-\ell(\sin p(\ell-b) \sin p b)(\sin p(\xi-b)-2 p \xi+p b+\sin p \xi \cos p b)- \\
& +\ell(\sin p(\ell-b))(p b \cos p b-\sin p b)(\cos p \xi \sin p b-2 \sin p \xi \cos p b)+ \\
& +b \ell(\sin p(\ell-b) \sin p b)(p-2 p \sin p \xi \sin p b-p \cos p \xi \cos p b)], \\
& c_{2 I}=\frac{1}{2 p^{3} \mathcal{D}_{c}}[(\sin p(\ell-b) \sin p b)(\sin p(\xi-b)-2 p \xi+p b+\sin p \xi \cos p b)+ \\
& +(\sin p(\ell-b))(\sin p b-p b \cos p b)(\cos p \xi \sin p b-2 \sin p \xi \cos p b)+ \\
& +b(\sin p b \sin p(\ell-b))(p \cos p \xi \cos p b-p+2 p \sin p \xi \sin p b)], \\
& c_{3 I}=\frac{1}{2 p^{3} \mathcal{D}_{c}}[(\ell-b)(\sin p \ell \sin p b)(\sin p(\xi-b)-2 p \xi+p b+\sin p \xi \cos p b)- \\
& (\ell-b)(\sin p \ell)(\sin p b-p b \cos b p)(\cos p \xi \sin p b-2 \sin p \xi \cos p b)+ \\
& +b(\ell-b)(\sin p b \sin p \ell)(p \cos p \xi \cos p b-p+2 p \sin p \xi \sin p b)], \\
& c_{4 I}=\frac{1}{2 p^{3} \mathcal{D}_{c}}[-(\ell-b) \cos p \ell \sin p b(\sin p(\xi-b)-2 p \xi+p b+\sin p \xi \cos b p)+ \\
& +(\ell-b) \cos p \ell(\sin p b-p b \cos p b)(\cos p \xi \sin p b-2 \sin p \xi \cos p b)- \\
& -b(\ell-b)(\cos p \ell \sin p b)(p \cos p \xi \cos p b-p+2 p \sin p \xi \sin p b)],
\end{aligned}
$$

in which

$$
\mathcal{D}_{c}=p b(\ell-b) \sin p \ell-\ell(\sin p b) \sin p(\ell-b) .
$$

With $a_{\ell I}, b_{\ell I}$ and $c_{\ell I}$

$$
G(x, \xi)= \begin{cases}G_{1 I}(x, \xi)=\sum_{\ell=1}^{4}\left(a_{\ell I}(\xi) \pm b_{\ell I}(\xi)\right) w_{\ell}(x) & \text { if } x \in[0, \ell] \\ G_{2 I}(x, \xi)=\sum_{\ell=1}^{4} c_{\ell I}(\xi) w_{\ell}(x) & \text { if } x \in[b, \ell] .\end{cases}
$$

is the Green function if $\xi \in[0, b]$. The sign is positive if $x<\xi$ and negative if $x>\xi$.

4.1.2. Green function if $\xi \in[b, \ell]$. The equation system for $b_{\ell I I}$ coincides with equation system 4.2. Hence $b_{\ell I I}=b_{\ell I}$ and the solutions are given by equation 4.3.

The boundary conditions in Table 1 for $x=0$ yield:

$$
c_{1 I I}=c_{3 I I}=0 \text {. }
$$

The continuity conditions at $x=b$ and the boundary conditions at $x=\ell$ lead to the following equation system - details of the calculation are all omitted:

$$
\left[\begin{array}{cccccc}
0 & 0 & 0 & 0 & b & \sin p b \\
1 & b & \cos p b & \sin p b & 0 & 0 \\
0 & 1 & -p \sin p b & p \cos p b & -1 & -p \cos p b \\
0 & 0 & -\cos p b & -\sin p b & 0 & \sin p b \\
1 & \ell & \cos p \ell & \sin p \ell & 0 & 0 \\
0 & 0 & -\cos p \ell & -\sin p \ell & 0 & 0
\end{array}\right]\left[\begin{array}{c}
a_{1 I I} \\
a_{2 I I} \\
a_{3 I I} \\
a_{4 I} \\
c_{2 I I} \\
c_{4 I I}
\end{array}\right]=
$$




$$
=\frac{1}{2 p^{3}}\left[\begin{array}{c}
0 \\
-p \xi+p b+\sin p(\xi-b) \\
p-p \cos p(\xi-b) \\
-\sin p(\xi-b) \\
p \xi-p \ell-\sin p(\xi-\ell) \\
\sin p(\xi-\ell)
\end{array}\right] .
$$

The solutions needed for calculating the Green function are gathered here:

$$
\begin{aligned}
& a_{1 I I}=\frac{1}{2 p^{3} \mathcal{D}_{c}}[(b p \ell \sin p \ell-\ell \sin p(\ell-b) \sin p b)(\sin p(\xi-b)-p \xi+p b)+ \\
& +(\sin p(\ell-\xi))\left(p b^{2} \sin p \ell-p b \ell \sin b p\right)-p b^{2}(\sin p \ell)(\sin p(\ell-\xi)-p \ell+p \xi)+ \\
& +\ell(\sin p(\xi-b) \sin p(\ell-b))(\sin p b-p b \cos p b)+ \\
& +b \ell(\sin p(\ell-b) \sin p b)(p-p \cos p(\xi-b))], \\
& a_{2 I I}=\frac{1}{2 p^{3} \mathcal{D}_{c}}[\sin p(\ell-\xi)(\sin p(\ell-b) \sin p b-p b(\sin p \ell-\sin p b))+ \\
& +(\sin p(\ell-b) \sin p b-p b \sin p \ell)(\sin p(\xi-b)-p \xi+p b)+ \\
& +(\sin p(\ell-b) \sin p b-p b \sin p \ell)(\sin p(\xi-\ell)-p \xi+p \ell)- \\
& -(\sin p(\xi-b) \sin p(\ell-b))(\sin p b-p b \cos p b)- \\
& -b(\sin p(\ell-b) \sin p b)(p-p \cos p(\xi-b))], \\
& a_{3 I I}=\frac{1}{2 p^{3} \mathcal{D}_{c}}[-b(\sin p \ell \sin p b)(\sin p(\xi-b)-p \xi+p b)- \\
& -(\sin p(\ell-\xi) \sin p b)(b \sin p \ell-\ell \sin p b)+ \\
& +b(\sin p \ell \sin p b)(\sin p(\ell-\xi)+p \xi-p \ell)- \\
& -(\sin p(\xi-b) \sin p \ell)(\sin p b-p b \cos p b)(\ell-b)- \\
& -b(\ell-b)(\sin p \ell \sin p b)(p-p \cos p(\xi-b))] \text {, } \\
& a_{4 I I}=\frac{1}{2 p^{3} \mathcal{D}_{c}}[\sin p(\ell-\xi)((\sin p b)(b \cos p \ell-\ell \cos p b)+p b(\ell-b))+ \\
& +b(\cos p \ell \sin p b)(\sin p(\xi-b)-p \xi+p b)+ \\
& +b(\cos p \ell \sin p b)(\sin p(\xi-\ell)-p \xi+p \ell)+ \\
& +(\sin p(\xi-b) \cos p \ell)(\sin p b-p b \cos p b)(\ell-b)+ \\
& +b(\ell-b)(\cos p \ell \sin p b)(p-p \cos p(\xi-b))], \\
& c_{1 I I}=0,
\end{aligned}
$$$$
c_{2 I I}=\frac{1}{2 p^{3} \mathcal{D}_{c}}[-(\sin p(\xi-\ell))(\sin p(\ell-b) \sin p b-p(\ell-b) \sin p b)+
$$$$
-\sin p(\xi-b)(\sin p(\ell-b) \sin p b-p(\ell-b) \cos p(\ell-b) \sin b p)-
$$$$
+(\sin p(\ell-b) \sin p b)(\sin p(\xi-\ell)-p \xi+p \ell)+
$$ 


$$
\begin{gathered}
+(\sin p(\ell-b) \sin p b)(\sin p(\xi-b)-p \xi+p b)+ \\
+(\ell-b)(\sin p(\ell-b) \sin p b)(p-p \cos p(\xi-b))], \\
c_{3 I I}=0 \\
c_{4 I I}=\frac{1}{2 p^{3} \mathcal{D}_{c}}[-b(\sin p(\ell-b))(\sin p(\xi-b)-p \xi+p b)+ \\
+\sin p(\xi-b)(b \sin p(\ell-b)-p b(\ell-b) \cos p(\ell-b))+ \\
+(\sin p(\xi-\ell))(b \sin p(\ell-b)-p b(\ell-b))- \\
-b(\sin p(\ell-b))(\sin p(\xi-\ell)-p \xi+p \ell)- \\
-b(\ell-b)(\sin p(\ell-b))(p-p \cos p(\xi-b))] .
\end{gathered}
$$

With $a_{\ell I I}, b_{\ell I I}$ and $c_{\ell I I}$

$$
G(x, \xi)= \begin{cases}G_{1 I I}(x, \xi)=\sum_{\ell=1}^{4} c_{\ell I}(\xi) w_{\ell}(x) & \text { if } x \in[0, b] \\ G_{2 I I}(x, \xi)=\sum_{\ell=1}^{4}\left(a_{\ell I I}(\xi) \pm b_{\ell I I}(\xi)\right) w_{\ell}(x) & \text { if } x \in[b, \ell] .\end{cases}
$$

is the Green function if $\xi \in[b, \ell]$. The sign is positive if $x<\xi$ and negative if $x>\xi$.

The Green function given by equations 4.9 and 4.11 satisfies the symmetry condition $G(x, \xi)=G(\xi, x), x, \xi \in[0, \ell]$. Fulfillment of the symmetry condition was checked numerically for a number of point pairs $x, \xi$.

4.2. PrsP beams subjected to a tensile force. If the axial force is tensile equation according to equation 2.5 (or 2.6a) equilibrium problems of PrsP beams are governed by the differential equation

$$
L[w(x)]=w^{(4)}-\mathcal{N} w^{(2)}=f_{z}
$$

for which the boundary and continuity conditions are presented in Table 1 .

4.2.1. Green function if $\xi \in[0, b]$. In the present subsection we shall apply the solution steps detailed in Subsection 4.1.1. Recalling that the general solution of the homogeneous differential equation $L[w(x)]=0$ is given by (2.6c) the continuity and discontinuity conditions 3.20 lead to the following equation system for $b_{\ell I}$ :

$$
\left[\begin{array}{cccc}
1 & \xi & \cosh p \xi & \sinh p \xi \\
0 & 1 & p \sinh p \xi & p \cosh p \xi \\
0 & 0 & p^{2} \cosh p \xi & p^{2} \sinh p \xi \\
0 & 0 & p^{3} \sinh p \xi & p^{3} \cosh p \xi
\end{array}\right]\left[\begin{array}{l}
b_{1} \\
b_{2} \\
b_{3} \\
b_{4}
\end{array}\right]=\left[\begin{array}{c}
0 \\
0 \\
0 \\
-\frac{1}{2}
\end{array}\right]
$$

from where

$$
\left[\begin{array}{l}
b_{1 I} \\
b_{2 I} \\
b_{3 I} \\
b_{4 I}
\end{array}\right]=\frac{1}{2 p^{3}}\left[\begin{array}{c}
-p \xi \\
p \\
\sinh p \xi \\
-\cosh p \xi
\end{array}\right]
$$

REMARK 4 . It has been taken again into account in the calculations that $w(x)$ corresponds to $y(x), k=2$ and $p_{2 k}=1$. 
We proceed with boundary and continuity conditions 3.23 . Recalling the boundary and continuity conditions in Table 1 and Remark 4. the equation system we obtain is formally the same as the equation system given by 4.4 , (4.5) and 4.6 the solution for $w$ is, however, different - see (2.6c). After substituting it we have

$$
\begin{aligned}
& a_{1 I}=-b_{1 I}=\frac{1}{2 p^{3}} p \xi \\
& a_{3 I}=-b_{3 I}=-\frac{1}{2 p^{3}} \sinh p \xi
\end{aligned}
$$

and

$$
\begin{gathered}
{\left[\begin{array}{cccccc}
b & \sinh p b & 0 & 0 & 0 & 0 \\
0 & 0 & 1 & b & \cosh p b & \sinh p b \\
1 & p \cosh p b & 0 & -1 & -p \sinh p b & -p \cosh p b \\
0 & \sinh p b & 0 & 0 & -\cosh p b & -\sinh p b \\
0 & 0 & 1 & \ell & \cosh p & \sinh p \ell \\
0 & 0 & 0 & 0 & \cosh p \ell & \sinh p \ell
\end{array}\right]\left[\begin{array}{c}
a_{2 I} \\
a_{4 I} \\
c_{1 I} \\
c_{2 I} \\
c_{3 I} \\
c_{4 i}
\end{array}\right]=} \\
=\frac{1}{2 p^{3}}\left[\begin{array}{c}
-2 p \xi+p b+\sinh (p \xi-b p)+\sinh p \xi \cosh p b \\
0 \\
p+2 p \sinh p \xi \sinh p b-p \cosh p \xi \cosh p b \\
2 \sinh p \xi \cos p b-\cosh p \xi \sinh p b \\
0 \\
0
\end{array}\right]
\end{gathered}
$$

from where

$$
\begin{aligned}
& a_{2 I}=\frac{1}{2 p^{3} \mathcal{D}_{t}} \times \\
& {[(p(\ell-b) \sinh p \ell-\sinh p b \sinh p(\ell-b))(p b-2 p \xi+\sinh p(\xi-b)+\sinh p \xi \cosh p b)-} \\
& -(\ell-b) \sinh p b \sinh p(\ell-b)(p+2 p \sinh p \xi \sinh p b-p \cosh p \xi \cosh p b)- \\
& -(p(\ell-b) \sinh p b \cosh p(\ell-b)-\sinh p b \sinh p(\ell-b)) \times \\
& (2 \sinh p \xi \cos p b-\cosh p \xi \sinh p b)], \\
& a_{4 I}= \\
& =\frac{1}{2 p^{3} \mathcal{D}_{t}}[-(\cosh p \xi \sinh p b-2 \sinh p \xi \cos p b)(b \sinh p(\ell-b)+p b(\ell-b) \cosh p(\ell-b))+ \\
& -(\ell-b)(\sinh p(\ell-b))(\sinh p(\xi-b)-2 p \xi+p b+\sinh p \xi \cosh p b)+ \\
& +b(\ell-b)(\sinh p(\ell-b))(p+2 p \sinh p \xi \sinh p b-p \cosh p \xi \cosh p b)] \text {, } \\
& c_{1 I}=\frac{1}{2 p^{3} \mathcal{D}_{t}}[\ell(\sinh p(\ell-b) \sinh p b)(\sinh p(\xi-b)-2 p \xi+p b+\sinh p \xi \cosh p b)- \\
& -\ell(\sinh p(\ell-b))(p b \cosh p b-\sinh p b)(\cosh p \xi \sinh p b-2 \sinh p \xi \cos p b)+ \\
& -b \ell(\sinh p(\ell-b) \sinh p b)(p+2 p \sinh p \xi \sinh p b-p \cosh p \xi \cosh p b)] \text {, }
\end{aligned}
$$




$$
\begin{aligned}
c_{2 I}= & \frac{1}{2 p^{3} \mathcal{D}_{t}}[-(\sinh p(\ell-b) \sinh p b)(\sinh p(\xi-b)-2 p \xi+p b+\sinh p \xi \cosh p b)+ \\
& -\sinh p(\ell-b)(\sinh p b-p b \cosh p b)(\cosh p \xi \sinh p b-2 \sinh p \xi \cos p b)- \\
& -b(\sinh p b \sinh p(\ell-b))(p \cosh p \xi \cosh p b-p-2 p \sinh p \xi \sinh p b)], \quad(4.15 \mathrm{~d}) \\
c_{3 I}= & \frac{1}{2 p^{3} \mathcal{D}_{t}}[-(\ell-b)(\sinh p \ell \sinh p b)(\sinh p(\xi-b)-2 p \xi+p b+\sinh p \xi \cosh p b)+ \\
- & (\ell-b)(\sinh p \ell)(\sinh p b-p b \cosh p b)(\cosh p \xi \sinh p b-2 \sinh p \xi \cos p b) \\
& -b(\ell-b)(\sinh p b \sinh p \ell)(p \cosh p \xi \cosh p b-p-2 p \sinh p \xi \sinh p b)], \quad(4.15 \mathrm{e}) \\
c_{4 I}= & \frac{1}{2 p^{3} \mathcal{D}}[(\ell-b)(\cosh p \ell \sinh p b)(\sinh p(\xi-b)-2 p \xi+p b+\sinh p \xi \cosh p b)+ \\
+ & (\ell-b) \cosh p \ell(\sinh p b-p b \cosh p b)(\cosh p \xi \sinh p b-2 \sinh p \xi \cos p b)+ \\
& +b(\ell-b)(\cosh p \ell \sinh p b)(p \cosh p \xi \cosh p b-p-2 p \sinh p \xi \sinh p b)] \quad(4.15 \mathrm{f})
\end{aligned}
$$

in which

$$
\mathcal{D}_{t}=b p(\ell-b) \sinh p \ell-\ell \sinh b p \sinh p(\ell-b) .
$$

With $a_{\ell I}, b_{\ell I}$ and $c_{\ell I}$ derived above for the case of the tensile axial force equation 4.9 can be used to find the Green function if $\xi \in[0, b]$.

4.2.2. Green function if $\xi \in[b, \ell]$. The equation system for $b_{\ell I I}$ coincides formally with equation system 4.13 . Consequently, $b_{\ell I I}=b_{\ell I}$ and the solutions are given by equation 4.14).

The boundary conditions in Table 1 for $x=0$ yield:

$$
c_{1 I I}=c_{3 I I}=0
$$

The continuity conditions at $x=b$ and the boundary conditions at $x=\ell$ lead to the following equation system - details of the calculation are all omitted:

$$
\begin{array}{r}
{\left[\begin{array}{cccccc}
0 & 0 & 0 & 0 & b & \sinh p b \\
1 & b & \cosh p b & \sinh p b & 0 & 0 \\
0 & 1 & p \sinh p b & p \cosh p b & -1 & -p \cosh p b \\
0 & 0 & \cosh p b & \sinh p b & 0 & -\sinh p b \\
1 & \ell & \cosh p \ell & \sinh p \ell & 0 & 0 \\
0 & 0 & \cosh p \ell & \sinh p \ell & 0 & 0
\end{array}\right]\left[\begin{array}{c}
a_{1 I I} \\
a_{2 I I} \\
a_{3 I I} \\
a_{4 I} \\
c_{2 I I} \\
c_{4 I I}
\end{array}\right]=} \\
=\frac{1}{2 p^{3}}\left[\begin{array}{c}
p \xi-p b-\sinh p(\xi-b) \\
-p+p \cosh p(\xi-b) \\
-\sinh p(\xi-b) \\
-p \xi+p \ell+\sinh p(\xi-\ell) \\
\sinh p(\xi-\ell)
\end{array}\right]
\end{array}
$$

The solutions obtained for $a_{1 I I}, \ldots, a_{4 I I}$ and $c_{1 I I}, \ldots, c_{4 I I}$ and needed for calculating the Green function are presented here: 


$$
\begin{aligned}
& a_{1 I I}=\frac{1}{2 p^{3} \mathcal{D}_{t}}[(p b \ell \sinh p \ell-\ell \sinh p b \sinh p(\ell-b))(-\sinh p(\xi-b)+p \xi-p b)+ \\
& +(\sinh p(\xi-\ell))\left(p b^{2} \sinh p \ell-p b \ell \sinh p b\right)-p b^{2}(\sinh p \ell)(\sinh p(\xi-\ell)+p \ell-p \xi)- \\
& -\ell(\sinh p(\xi-b) \sinh p(\ell-b))(\sinh p b-p b \cosh p b)- \\
& -b \ell(\sinh p(\ell-b) \sinh p b)(p-p \cosh p(\xi-b))], \\
& a_{2 I I}=\frac{1}{2 p^{3} \mathcal{D}}[-\sinh p(\ell-\xi)(\sinh p(\ell-b) \sinh p b+p b(-\sinh p \ell+\sinh p b))+ \\
& +(\sinh p(\ell-b) \sinh p b-p b \sinh p \ell)(-\sinh p(\xi-b)+p \xi-p b)- \\
& -(\sinh p(\ell-b) \sinh p b-p b \sinh p \ell)(\sinh p(\xi-\ell)+p \ell-p \xi)+ \\
& +(\sinh p(\xi-b) \sinh p(\ell-b))(\sinh p b-p b \cosh p b)+ \\
& +b(\sinh p b \sinh p(\ell-b))(p-p \cosh p(\xi-b))], \\
& a_{3 I I}=\frac{1}{2 p^{3} \mathcal{D}_{t}}[b(\sinh p \ell \sinh p b)(\sinh p(\xi-b)-p \xi+p b)- \\
& -(\sinh p(\ell-\xi) \sinh p b)(b \sinh p \ell-\ell \sinh p b)- \\
& -b(\sinh p \ell \sinh p b)(\sinh p(\ell-\xi)+p \xi-p \ell)- \\
& -(\sinh p(\xi-b) \sinh p \ell)(\sinh p b-p b \cosh p b)(\ell-b)- \\
& -b(\ell-b)(\sinh p \ell \sinh p b)(p-p \cosh p(\xi-b))], \\
& a_{4 I I}=\frac{1}{2 p^{3} \mathcal{D}_{t}}[-\sinh p(\ell-\xi)((\sinh p b)(b \cosh p \ell-\ell \cosh p b)+p b(\ell-b))- \\
& -b(\cosh p \ell \sinh p b)(\sinh p(\xi-b)-p \xi+p b)- \\
& -b(\cosh p \ell \sinh p b)(\sinh p(\xi-\ell)-p \xi+p \ell)- \\
& -(\sinh p(\xi-b) \cosh p \ell)(\sinh p b-p b \cosh p b)(\ell-b)- \\
& -b(\ell-b)(\cosh p \ell \sinh p b)(p-p \cosh p(\xi-b))], \\
& c_{1 I I}=0, \\
& -(\sinh p(\ell-b) \sinh p b)(\sinh p(\xi-b)-p \xi+p b)+ \\
& -(\ell-b)(\sinh p(\ell-b) \sinh p b)(p-p \cosh p(\xi-b))], \\
& c_{3 I I}=0,
\end{aligned}
$$




$$
\begin{aligned}
& -(\sinh p(\xi-\ell))(b \sinh p(\ell-b)-p b(\ell-b))+ \\
& +b(\sinh p(\ell-b))(\sinh p(\xi-\ell)-p \xi+p \ell)+ \\
& +b(\ell-b)(\sinh p(\ell-b))(p-p \cosh p(\xi-b))] .
\end{aligned}
$$

With $a_{\ell I I}, b_{\ell I I}=b_{\ell I}$ and $c_{\ell I}$ presented above for the tensile axial force equation 4.11 can be used to find the Green function if $\xi \in[0, b]$. According to a numerical check the Green function satisfies the symmetry condition $G(x, \xi)=G(\xi, x)$.

4.2.3. Graphical representation of the Green function. The dimensionless critical force $\mathcal{N}_{\text {crit }}\left(p_{\text {crit }}\right)$ as function of the location of the intermediate roller support is given by the polynomial [17]:

$$
\begin{aligned}
& \sqrt{\mathcal{N}_{\text {crit }}} / \pi=p_{\text {crit }} / \pi= \\
& =-25.6511182911 b^{5}+18.3289788300 b^{4}-4.70118403874 b^{3}+ \\
& +1.39533914036 b^{2}+0.928843353123 b+1.43050335236, \quad b \in[0,0.5] .
\end{aligned}
$$

Note that for symmetry reasons $b \in[0,0.5]$.

Assume that $b=0.5$ and $\xi=0.75$. Assume further that the axial force is compressive. Figure 3 shows the Green function provided that $p=0.4 p_{\text {crit }}$ and $p=0.8 p_{\text {crit }}$. Since the Green function is the dimensionless vertical displacement due to a dimensionless unit force applied to the beam at $\xi=0.75$ it follows that the bending moment caused by the compressive force $\mathcal{N}$ has the same sign as the bending moment caused by the dimensionless unit force. Its magnitude increases with $\mathcal{N}$. The same is valid for the magnitude of the Green function. Figure 3 clearly shows this change.

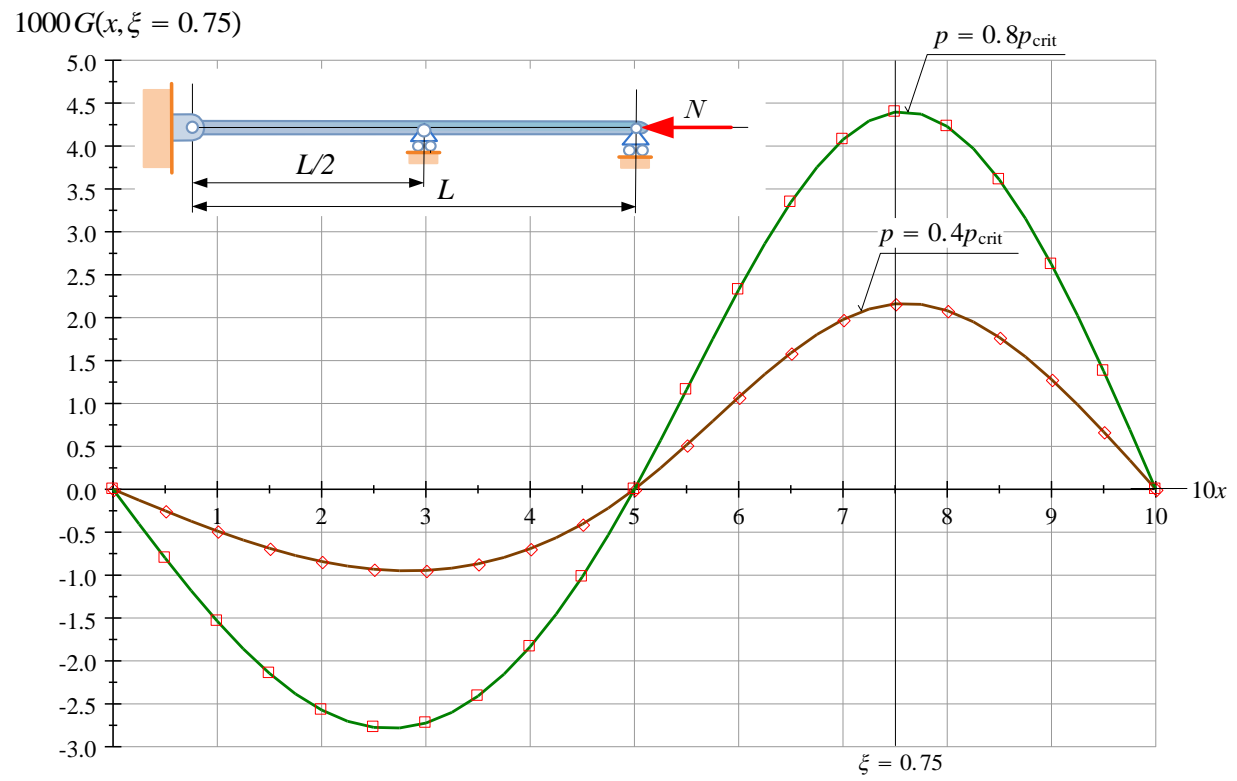

Figure 3. 


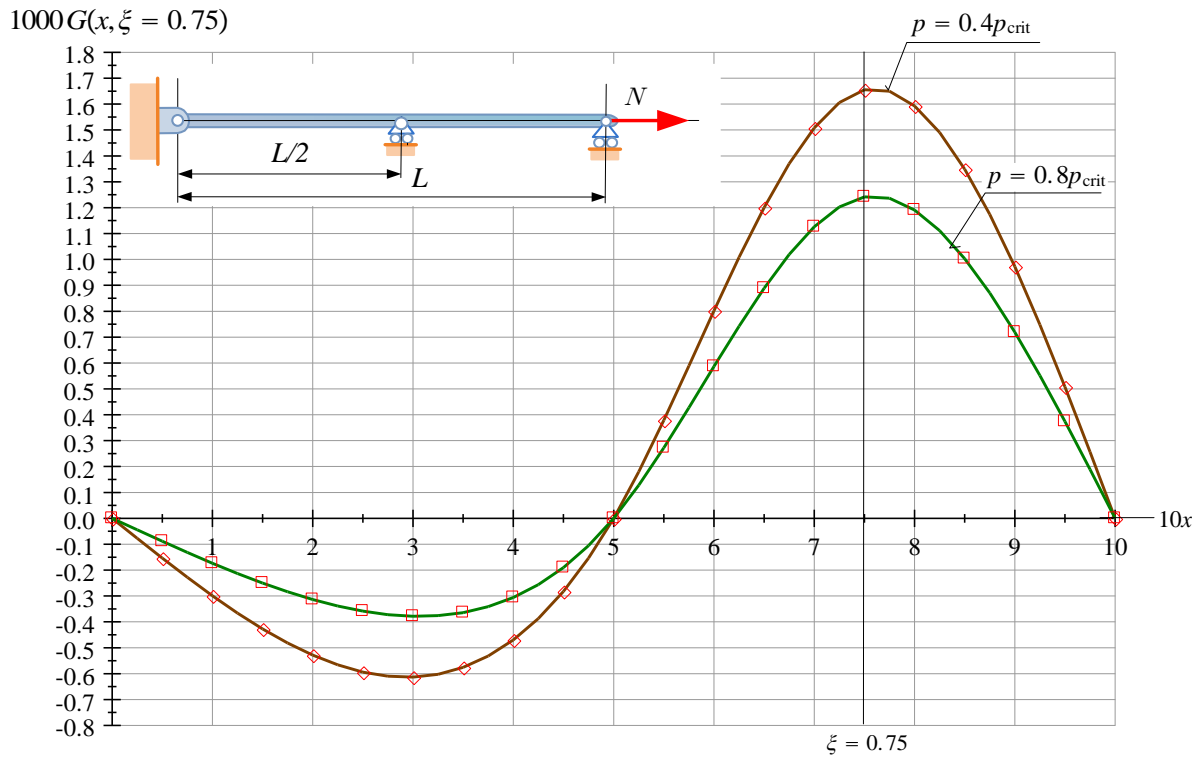

Figure 4.

Assume again that $b=0.5$ and $\xi=0.75$ but the axial force is tensile. Figure 4 shows the Green function provided that $p=0.4 p_{\text {crit }}$ and $p=0.8 p_{\text {crit }}$. This time there is a sign difference between the bending moments due to the dimensionless unit force and the tensile force. Hence the magnitude of the Green function decreases as the axial force $\mathcal{N}$ increases. Figure 4 clearly represents this phenomenon.

According to equation (3.4) the dimensionless vertical displacement $w$ due to the dimensionless vertical force system $f_{z}$ can be calculated by performing the integral in

$$
w(x)=\int_{\xi=0}^{\ell=1} G(x, \xi) f_{z}(\xi) \mathrm{d} \xi .
$$

\section{Computational Results}

5.1. Integral equation of the problem. We shall assume that the inertia forces resulting from the longitudinal motion in the axial direction are neglected. It is also assumed that the moments of the inertia forces obtained from the rotation of cross section are negligible. Under these assumptions the dimensionless amplitude of the vibrations is denoted by $w$. It can be seen on the basis of Subsection 8.16 in [18, (4.1) and 4.12 that the amplitude $w$ should fulfill the differential equation

$$
\begin{aligned}
& L[w(x)]=w^{(4)} \pm \mathcal{N} w^{(2)}=\lambda w=f_{z}, \\
& p^{2}=\mathcal{N}=\frac{L^{2} N}{I_{e y}}, \quad \lambda=\frac{\rho_{a} A \omega^{2} L^{4}}{I_{e y}},
\end{aligned}
$$

where $\rho_{a}$ is the average density over the cross section of the beam, $\omega$ is the natural circular frequency while $\lambda$ is the eigenvalue sought. Differential equation (5.1) is 
associated with the homogeneous boundary and continuity conditions presented in Table 1. These constitute an eigenvalue problem with $\lambda$ as the eigenvalue. If we substitute $\lambda w(\xi)$ for $f_{z}(\xi)$ in 4.19 we obtain the homogeneous Fredholm integral equation

$$
w(x)=\lambda \int_{\xi=0}^{\ell=1} G(x, \xi) w(\xi) \mathrm{d} \xi
$$

for which the kernel $G(x, \xi)$ is presented in Subsection 4.1 if the axial force is compressive and in Subsection 4.2 if the axial force is tensile. The above integral equation determines an eigenvalue problem equivalent to the eigenvalue problem determined by differential equation (5.1) and the homogeneous boundary and continuity conditions presented in Table 1. Making use of the boundary element algorithm presented in [15 - see Subsection 7.2 - the eigenvalue problem determined by $(5.2)$ can be reduced to an algebraic eigenvalue problem which can be solved numerically. A Fortran 90 code has been developed and applied to find numerical solutions for the eigenvalue $\lambda$. The interval $[0, \ell=1]$ was divided into 12 elements and a quadratic isoparametric approximation was used over the elements in the code we developed.

If the axial force is zero the lowest dimensionless eigenvalue and circular frequency will be denoted by $\check{\lambda}_{1}$ and $\check{\omega}_{1}$.

5.2. Numerical results if $b$ tends to zero. Table 2 represents the computational results if $b \longrightarrow 0$. The quotient $\sqrt{\mathcal{N}_{\text {crit }}} / \pi$ is computed using equation (4.18). The value of $\check{\lambda}_{1}$ is given by equation (105) in 15 :

$$
\begin{aligned}
\sqrt{\check{\lambda}_{1}} / \pi^{2}= & 402.66423 \times \\
+2494.995 \times & (1.0-b)^{5}-1594.73367 \times(1.0-b)^{4}+ \\
& +716.4361 \times(1.0-b)-99.3904, \quad b \in[0,0.5],
\end{aligned}
$$

or can be taken from Table 2 in 15. This is also valid for Tables 3 ....7 which have the same structure as Table 2. The quotient $\omega_{1}^{2} / \check{\omega}_{1}^{2}=\lambda_{1} / \check{\lambda}_{1}$ is computed for $\mathcal{N} / \mathcal{N}_{\text {crit }}=0.00,0.10, \ldots, 0.90-$ see columns 2,3 and 5 in Tables $2, \ldots .7$.

The computed values of $\omega_{1}^{2} / \check{\omega}_{1}^{2}=\lambda_{1} / \check{\lambda}_{1}$ are denoted by diamonds in Figures $5, \ldots .9$. The difference between two subsequent values of $\omega_{1}^{2} / \check{\omega}_{1}^{2}$ is also included in these tables - see columns 4 and 6 . If the function $\omega_{1}^{2} / \check{\omega}_{1}^{2}\left(\mathcal{N} / \mathcal{N}_{\text {crit }}\right)$ is [non-linear](linear) the difference [varies] (is constant).

Quadratic polynomials are fitted onto the computational results. Their graphs are drawn using continuous lines in the figures.

The beam behaves as if it were a fixed-pinned beam if $b \longrightarrow 0$. Hence the results obtained should be the same as those valid for fixed-pinned beams. A comparison of the present results to those published in 18 - see Section 8.17.2 - proves that there is a very good agreement. 
Table 2 .

\begin{tabular}{|c|c|c|c|c|c|}
\cline { 3 - 6 } \multicolumn{2}{c|}{} & \multicolumn{4}{|c|}{$b$ tends to zero } \\
\cline { 3 - 6 } \multicolumn{2}{c|}{} & \multicolumn{3}{|c|}{$\sqrt{\mathcal{N}_{\text {crit }}} / \pi=1.4304, \check{\lambda}_{1}=1.5622$} \\
\cline { 3 - 6 } \multicolumn{2}{c|}{ Compression } & \multicolumn{2}{c|}{ Tension } \\
\hline $\begin{array}{c}\text { Load } \\
\text { step }\end{array}$ & $\begin{array}{c}\mathcal{N} / \mathcal{N}_{\text {crit }} \\
\left(p^{2} / p_{\text {crit }}^{2}\right)\end{array}$ & $\begin{array}{c}\omega_{1}^{2} / \check{\omega}_{1}^{2} \\
\left(\lambda_{1} / \check{\lambda}_{1}\right)\end{array}$ & Difference & $\begin{array}{c}\omega_{1}^{2} / \check{\omega}_{1}^{2} \\
\left(\lambda_{1} / \check{\lambda}_{1}\right)\end{array}$ & Difference \\
\hline \hline 1 & 0.00 & 1.000000 & & 1.000000 & \\
\hline 2 & 0.10 & 0.902010 & 0.0979900 & 1.097670 & 0.097670 \\
\hline 3 & 0.20 & 0.803642 & -0.098369 & 1.195005 & 0.097335 \\
\hline 4 & 0.30 & 0.704879 & -0.098763 & 1.292038 & 0.097032 \\
\hline 5 & 0.40 & 0.605692 & -0.099186 & 1.388785 & 0.096747 \\
\hline 6 & 0.50 & 0.506050 & -0.099642 & 1.485264 & 0.096478 \\
\hline 7 & 0.60 & 0.405916 & -0.100134 & 1.581488 & 0.096224 \\
\hline 8 & 0.70 & 0.305247 & -0.100668 & 1.677471 & 0.095983 \\
\hline 9 & 0.80 & 0.203999 & -0.101248 & 1.773227 & 0.095755 \\
\hline 10 & 0.90 & 0.102117 & -0.101881 & 1.868766 & 0.095538 \\
\hline
\end{tabular}

$\omega_{1}^{2} / \breve{\omega}_{1}^{2} \quad b \rightarrow 0$

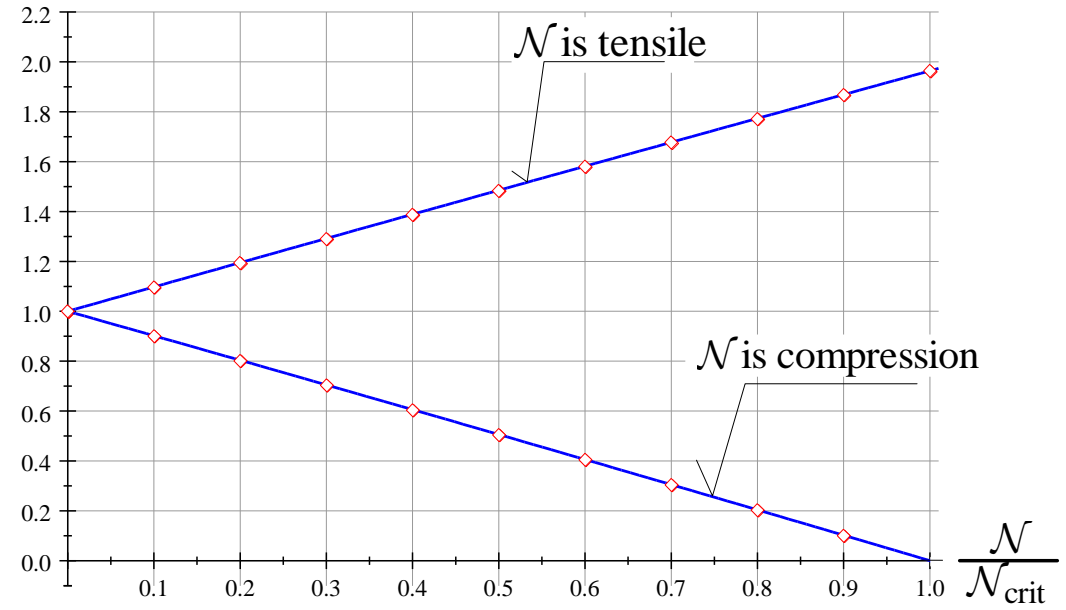

Figure 5.

The quadratic polynomials fitted onto the computational results both for compression and tension are given by the following equations:

$$
\begin{aligned}
& \frac{\omega_{1}^{2}}{\check{\omega}_{1}^{2}}=\frac{\lambda_{1}}{\check{\lambda}_{1}}=0.9999-0.9756 \frac{\mathcal{N}}{\mathcal{N}_{\text {crit }}}-2.4061 \times 10^{-2}\left(\frac{\mathcal{N}}{\mathcal{N}_{\text {crit }}}\right)^{2}, \\
& \frac{\omega_{1}^{2}}{\check{\omega}_{1}^{2}}=\frac{\lambda_{1}}{\check{\lambda}_{1}}=1.0000+0.9770 \frac{\mathcal{N}}{\mathcal{N}_{\text {crit }}}-1.3205 \times 10^{-2}\left(\frac{\mathcal{N}}{\mathcal{N}_{\text {crit }}}\right)^{2}
\end{aligned}
$$


5.3. Numerical results if $b=0.1$. Table 3 and Figure 6 represent the results obtained.

Table 3.

\begin{tabular}{|c|c|c|c|c|c|}
\cline { 3 - 6 } \multicolumn{2}{c|}{} & \multicolumn{4}{|c|}{$b=0.1$} \\
\cline { 3 - 6 } \multicolumn{2}{c|}{} & \multicolumn{3}{|c|}{$\sqrt{\mathcal{N}_{\text {crit }}} / \pi=1.5341, \check{\lambda}_{1}=1.8098$} \\
\cline { 3 - 6 } & \multicolumn{2}{|c|}{ Compression } & \multicolumn{2}{c|}{ Tension } \\
\hline $\begin{array}{r}\text { Load } \\
\text { step }\end{array}$ & $\begin{array}{c}\mathcal{N} / \mathcal{N}_{\text {crit }} \\
\left(p^{2} / p_{\text {crit }}^{2}\right)\end{array}$ & $\begin{array}{c}\omega_{1}^{2} / \check{\omega}_{1}^{2} \\
\left(\lambda_{1} / \check{\lambda}_{1}\right)\end{array}$ & Difference & $\begin{array}{l}\omega_{1}^{2} / \check{\omega}_{1}^{2} \\
\left(\lambda_{1} / \check{\lambda}_{1}\right)\end{array}$ & Difference \\
\hline \hline 1 & 0.00 & 1.000000 & & 1.000000 & \\
\hline 2 & 0.10 & 0.898254 & -0.098720 & 1.093780 & 0.093780 \\
\hline 3 & 0.20 & 0.800038 & -0.098215 & 1.191130 & 0.097350 \\
\hline 4 & 0.30 & 0.701491 & -0.098547 & 1.288230 & 0.097099 \\
\hline 5 & 0.40 & 0.602586 & -0.098905 & 1.385094 & 0.096863 \\
\hline 6 & 0.50 & 0.503293 & -0.099292 & 1.481736 & 0.096642 \\
\hline 7 & 0.60 & 0.403580 & -0.099712 & 1.578170 & 0.096434 \\
\hline 8 & 0.70 & 0.303411 & -0.100169 & 1.674408 & 0.096237 \\
\hline 9 & 0.80 & 0.202743 & -0.100667 & 1.770460 & 0.096051 \\
\hline 10 & 0.90 & 0.101529 & -0.101214 & 1.866336 & 0.095876 \\
\hline
\end{tabular}

$\omega_{1}^{2} / \check{\omega}_{1}^{2} \quad b=0.1$

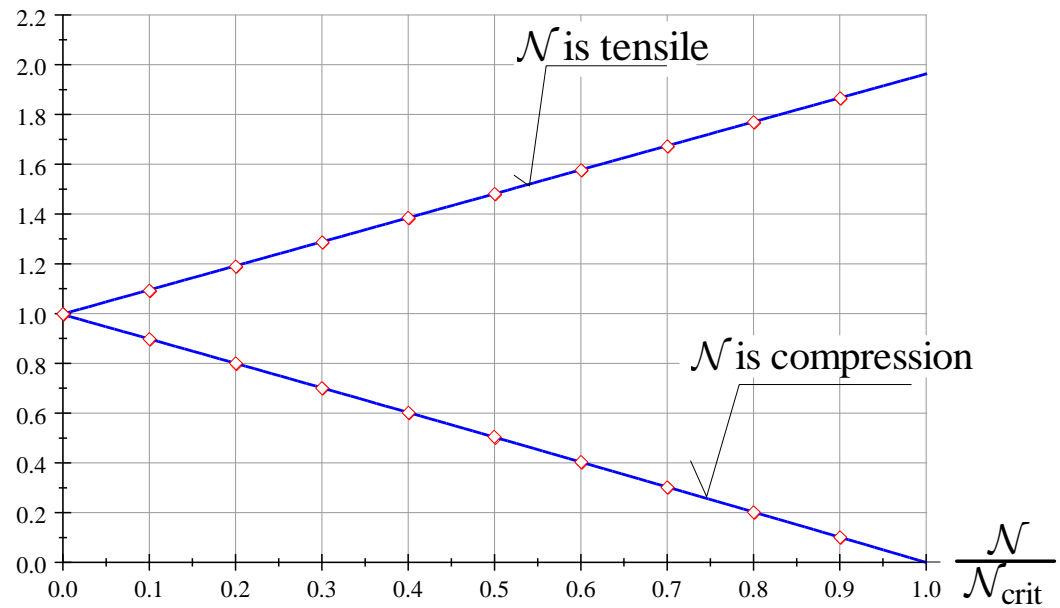

Figure 6.

The quadratic polynomials fitted onto the numerical results are as follows:

$$
\begin{aligned}
& \frac{\omega_{1}^{2}}{\check{\omega}_{1}^{2}}=\frac{\lambda_{1}}{\check{\lambda}_{1}}=0.998400-0.985245 \frac{\mathcal{N}}{\mathcal{N}_{\text {crit }}}-1.174606 \times 10^{-2}\left(\frac{\mathcal{N}}{\mathcal{N}_{\text {crit }}}\right)^{2}, \\
& \frac{\omega_{1}^{2}}{\check{\omega}_{1}^{2}}=\frac{\lambda_{1}}{\check{\lambda}_{1}}=0.998592+0.966543 \frac{\mathcal{N}}{\mathcal{N}_{\text {crit }}}-2.109042 \times 10^{-3}\left(\frac{\mathcal{N}}{\mathcal{N}_{\text {crit }}}\right)^{2} .
\end{aligned}
$$


5.4. Numerical results if $b=0.2$. Table 4 and Figure 6 represent the results obtained.

Table 4.

\begin{tabular}{|c|c|c|l|l|l|}
\cline { 3 - 6 } \multicolumn{2}{c|}{} & \multicolumn{4}{|c|}{$b=0.2$} \\
\cline { 3 - 6 } \multicolumn{2}{c|}{} & \multicolumn{3}{|c|}{$\sqrt{\mathcal{N}_{\text {crit }} / \pi=1.6557, \check{\lambda}_{1}=2.1610}$} \\
\cline { 3 - 6 } \multicolumn{2}{c|}{} & \multicolumn{2}{c|}{ Compression } & \multicolumn{2}{c|}{ Tension } \\
\hline $\begin{array}{r}\text { Load } \\
\text { step }\end{array}$ & $\begin{array}{c}\mathcal{N} / \mathcal{N}_{\text {crit }} \\
\left(p^{2} / p_{\text {crit }}^{2}\right)\end{array}$ & $\begin{array}{c}\omega_{1}^{2} / \check{\omega}_{1}^{2} \\
\left(\lambda_{1} / \check{\lambda}_{1}\right)\end{array}$ & Difference & $\begin{array}{l}\omega_{1}^{2} / \check{\omega}_{1}^{2} \\
\left(\lambda_{1} / \check{\lambda}_{1}\right)\end{array}$ & Difference \\
\hline \hline 1 & 0.00 & 1.000000 & & 1.000000 & \\
\hline 2 & 0.10 & 0.904873 & -0.099724 & 1.104529 & 0.099631 \\
\hline 3 & 0.20 & 0.804869 & -0.100004 & 1.204192 & 0.099662 \\
\hline 4 & 0.30 & 0.704740 & -0.100128 & 1.303750 & 0.099558 \\
\hline 5 & 0.40 & 0.604481 & -0.100259 & 1.403208 & 0.099458 \\
\hline 6 & 0.50 & 0.504084 & -0.100396 & 1.502570 & 0.099362 \\
\hline 7 & 0.60 & 0.403543 & -0.100541 & 1.601840 & 0.099269 \\
\hline 8 & 0.70 & 0.302849 & -0.100693 & 1.701020 & 0.099180 \\
\hline 9 & 0.80 & 0.201995 & -0.100854 & 1.800115 & 0.099094 \\
\hline 10 & 0.90 & 0.100970 & -0.101025 & 1.899127 & 0.099011 \\
\hline
\end{tabular}

$\omega_{1}^{2} / \check{\omega}_{1}^{2} \quad b=0.2$

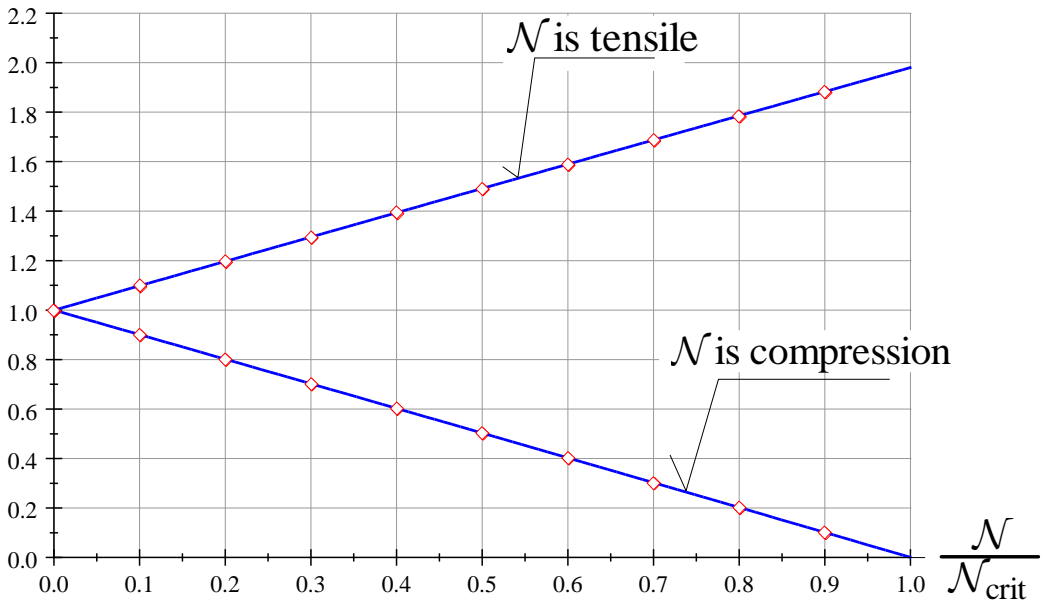

Figure 7.

Equations

$$
\begin{aligned}
& \frac{\omega_{1}^{2}}{\check{\omega}_{1}^{2}}=\frac{\lambda_{1}}{\check{\lambda}_{1}}=0.999596-0.987083 \frac{\mathcal{N}}{\mathcal{N}_{\text {crit }}}-1.214649 \times 10^{-2}\left(\frac{\mathcal{N}}{\mathcal{N}_{\text {crit }}}\right)^{2}, \\
& \frac{\omega_{1}^{2}}{\check{\omega}_{1}^{2}}=\frac{\lambda_{1}}{\check{\lambda}_{1}}=0.999633+0.987212 \frac{\mathcal{N}}{\mathcal{N}_{\text {crit }}}-6.295185 \times 10^{-3}\left(\frac{\mathcal{N}}{\mathcal{N}_{\text {crit }}}\right)^{2} .
\end{aligned}
$$

are the quadratic polynomials fitted onto the computational results. 
5.5. Numerical results if $b=0.3$. Table 5 . Figure 8 and equations (5.7) represent the results obtained.

Table 5.

\begin{tabular}{|c|c|c|c|c|c|}
\hline & \multicolumn{4}{|c|}{$b=0.3$} \\
\hline & & \multicolumn{4}{|c|}{$\sqrt{\mathcal{N}_{\text {crit }}} / \pi=1.79382, \check{\lambda}_{1}=2.6683$} \\
\hline & & \multicolumn{2}{|c|}{ Compression } & \multicolumn{2}{|c|}{ Tension } \\
\hline $\begin{array}{r}\text { Load } \\
\text { step }\end{array}$ & $\begin{array}{c}\mathcal{N} / \mathcal{N}_{\text {crit }} \\
\left(p^{2} / p_{\text {crit }}^{2}\right)\end{array}$ & $\begin{array}{c}\omega_{1}^{2} / \check{\omega}_{1}^{2} \\
\left(\lambda_{1} / \check{\lambda}_{1}\right)\end{array}$ & Difference & $\begin{array}{l}\omega_{1}^{2} / \check{\omega}_{1}^{2} \\
\left(\lambda_{1} / \check{\lambda}_{1}\right)\end{array}$ & Difference \\
\hline 1 & 0.00 & 1.000000 & & 1.000000 & \\
\hline 2 & 0.10 & 0.900657 & -0.099075 & 1.098279 & 0.098279 \\
\hline 3 & 0.20 & 0.801574 & -0.099083 & 1.196842 & 0.098563 \\
\hline 4 & 0.30 & 0.702290 & -0.099283 & 1.295255 & 0.098412 \\
\hline 5 & 0.40 & 0.602790 & -0.099500 & 1.393526 & 0.098271 \\
\hline 6 & 0.50 & 0.503055 & -0.099734 & 1.491664 & 0.098138 \\
\hline 7 & 0.60 & 0.403065 & -0.099990 & 1.589678 & 0.098013 \\
\hline 8 & 0.70 & 0.302795 & -0.100269 & 1.687574 & 0.097895 \\
\hline 9 & 0.80 & 0.202221 & -0.100574 & 1.785359 & 0.097784 \\
\hline 9 & 0.90 & 0.101311 & -0.100910 & 1.883038 & 0.097679 \\
\hline
\end{tabular}

$\omega_{1}^{2} / \check{\omega}_{1}^{2} \quad b=0.3$

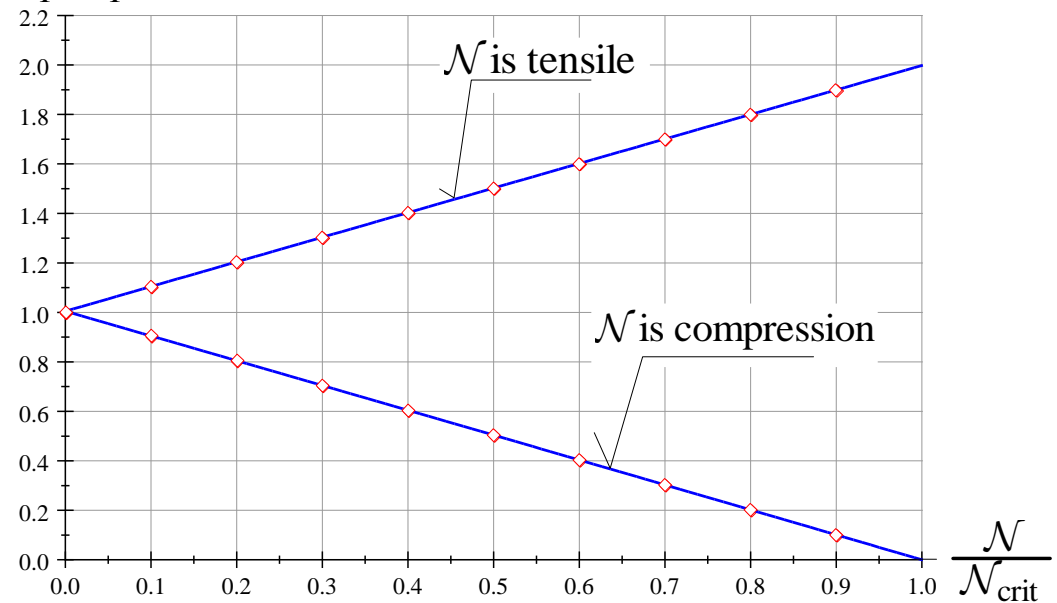

Figure 8.

$$
\begin{aligned}
& \frac{\omega_{1}^{2}}{\check{\omega}_{1}^{2}}=\frac{\lambda_{1}}{\check{\lambda}_{1}}=1.004689-0.997570 \frac{\mathcal{N}}{\mathcal{N}_{\text {crit }}}-7.276534 \times 10^{-3}\left(\frac{\mathcal{N}}{\mathcal{N}_{\text {crit }}}\right)^{2}, \\
& \frac{\omega_{1}^{2}}{\check{\omega}_{1}^{2}}=\frac{\lambda_{1}}{\check{\lambda}_{1}}=1.004794+0.997875 \frac{\mathcal{N}}{\mathcal{N}_{\text {crit }}}-4.643405 \times 10^{-3}\left(\frac{\mathcal{N}}{\mathcal{N}_{\text {crit }}}\right)^{2} .
\end{aligned}
$$


5.6. Numerical results if $b=0.4$. Table 6 and Figure 9 represent the results obtained.

Table 6 .

\begin{tabular}{|c|c|c|c|c|c|}
\cline { 3 - 6 } \multicolumn{2}{c|}{} & \multicolumn{4}{|c|}{$b=0.4$} \\
\cline { 3 - 6 } \multicolumn{2}{c|}{} & \multicolumn{3}{|c|}{$\sqrt{\mathcal{N}_{\text {crit }}} / \pi=1.93113, \check{\lambda}_{1}=3.3881$} \\
\cline { 3 - 6 } & \multicolumn{2}{|c|}{ Compression } & \multicolumn{2}{c|}{ Tension } \\
\hline $\begin{array}{r}\text { Load } \\
\text { step }\end{array}$ & $\begin{array}{c}\mathcal{N} / \mathcal{N}_{\text {crit }} \\
\left(p^{2} / p_{\text {crit }}^{2}\right)\end{array}$ & $\begin{array}{c}\omega_{1}^{2} / \check{\omega}_{1}^{2} \\
\left(\lambda_{1} / \check{\lambda}_{1}\right)\end{array}$ & Difference & $\begin{array}{l}\omega_{1}^{2} / \check{\omega}_{1}^{2} \\
\left(\lambda_{1} / \check{\lambda}_{1}\right)\end{array}$ & Difference \\
\hline \hline 1 & 0.00 & 1.000000 & & 1.000000 & \\
\hline 2 & 0.10 & 0.894987 & -0.098455 & 1.091328 & 0.098059 \\
\hline 3 & 0.20 & 0.796529 & -0.098458 & 1.189232 & 0.097904 \\
\hline 4 & 0.30 & 0.697862 & -0.098666 & 1.286971 & 0.097739 \\
\hline 5 & 0.40 & 0.598974 & -0.098888 & 1.384555 & 0.097583 \\
\hline 6 & 0.50 & 0.499848 & -0.099125 & 1.481990 & 0.097435 \\
\hline 7 & 0.60 & 0.400468 & -0.099380 & 1.579285 & 0.097294 \\
\hline 8 & 0.70 & 0.300814 & -0.099653 & 1.676446 & 0.097160 \\
\hline 9 & 0.80 & 0.200866 & -0.099947 & 1.773479 & 0.097033 \\
\hline 9 & 0.90 & 0.100599 & -0.100266 & 1.870391 & 0.096911 \\
\hline
\end{tabular}

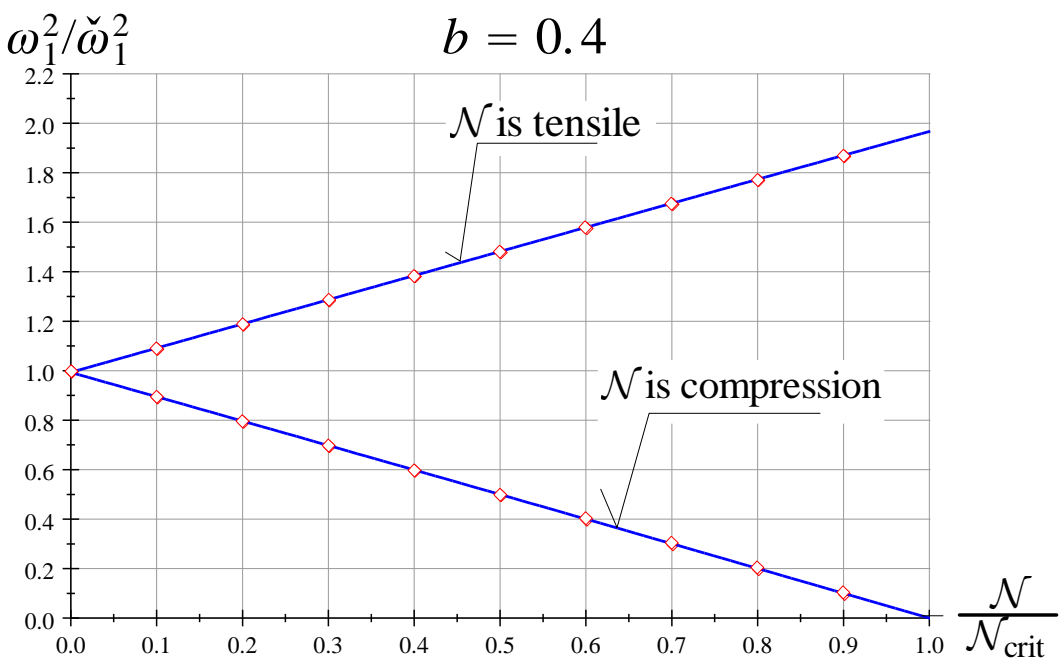

Figure 9 .

The two quadratic polynomials fitted onto the numerical results are presented here:

$$
\begin{aligned}
& \frac{\omega_{1}^{2}}{\check{\omega}_{1}^{2}}=\frac{\lambda_{1}}{\check{\lambda}_{1}}=0.993088-0.980042 \frac{\mathcal{N}}{\mathcal{N}_{\text {crit }}}-1.286556 \times 10^{-2}\left(\frac{\mathcal{N}}{\mathcal{N}_{\text {crit }}}\right)^{2}, \\
& \frac{\omega_{1}^{2}}{\check{\omega}_{1}^{2}}=\frac{\lambda_{1}}{\check{\lambda}_{1}}=0.993323+0.980873 \frac{\mathcal{N}}{\mathcal{N}_{\text {crit }}}-7.0740713 \times 10^{-3}\left(\frac{\mathcal{N}}{\mathcal{N}_{\text {crit }}}\right)^{2} .
\end{aligned}
$$


5.7. Numerical results if $b=0.5$. Table 7 represents the results obtained.

Table 7.

\begin{tabular}{|c|c|c|c|c|c|}
\cline { 3 - 6 } \multicolumn{2}{c|}{} & \multicolumn{4}{|c|}{$b=0.5$} \\
\cline { 3 - 6 } \multicolumn{2}{c|}{} & \multicolumn{3}{|c|}{$\sqrt{\mathcal{N}_{\text {crit }}} / \pi=2.0000, \check{\lambda}_{1}=4.0000$} \\
\cline { 3 - 6 } \multicolumn{2}{c|}{ Compression } & \multicolumn{2}{c|}{ Tension } \\
\hline $\begin{array}{r}\text { Load } \\
\text { step }\end{array}$ & $\begin{array}{c}\mathcal{N} / \mathcal{N}_{\text {crit }} \\
\left(p^{2} / p_{\text {crit }}^{2}\right)\end{array}$ & $\begin{array}{c}\omega_{1}^{2} / \check{\omega}_{1}^{2} \\
\left(\lambda_{1} / \check{\lambda}_{1}\right)\end{array}$ & Difference & $\begin{array}{l}\omega_{1}^{2} / \check{\omega}_{1}^{2} \\
\left(\lambda_{1} / \check{\lambda}_{1}\right)\end{array}$ & Difference \\
\hline \hline 1 & 0.00 & 1.000000 & & 1.000000 & \\
\hline 2 & 0.10 & 0.893682 & -0.099310 & 1.092295 & 0.099313 \\
\hline 3 & 0.20 & 0.794376 & -0.099306 & 1.191601 & 0.099306 \\
\hline 4 & 0.30 & 0.695069 & -0.099306 & 1.290908 & 0.099306 \\
\hline 5 & 0.40 & 0.595763 & -0.099306 & 1.390214 & 0.099306 \\
\hline 6 & 0.50 & 0.496457 & -0.099306 & 1.489521 & 0.099306 \\
\hline 7 & 0.60 & 0.397150 & -0.099306 & 1.588827 & 0.099306 \\
\hline 8 & 0.70 & 0.297844 & -0.099306 & 1.688133 & 0.099306 \\
\hline 9 & 0.80 & 0.198538 & -0.099306 & 1.787440 & 0.099306 \\
\hline 9 & 0.90 & 0.099231 & -0.099306 & 1.886746 & 0.099306 \\
\hline
\end{tabular}

The polynomial approximations (the differences are constants)

$$
\begin{aligned}
& \frac{\omega_{1}^{2}}{\check{\omega}_{1}^{2}}=\frac{\lambda_{1}}{\check{\lambda}_{1}}=0.993064-0.992989 \frac{\mathcal{N}}{\mathcal{N}_{\text {crit }}} \approx 1.000-\frac{\mathcal{N}}{\mathcal{N}_{\text {crit }}}, \\
& \frac{\omega_{1}^{2}}{\check{\omega}_{1}^{2}}=\frac{\lambda_{1}}{\check{\lambda}_{1}}=0.993064+0.992989 \frac{\mathcal{N}}{\mathcal{N}_{\text {crit }}} \approx 1.000+\frac{\mathcal{N}}{\mathcal{N}_{\text {crit }}} .
\end{aligned}
$$

show that the functions $\omega_{1}^{2} / \check{\omega}_{1}^{2}\left(\mathcal{N} / \mathcal{N}_{\text {crit }}\right)$ fitted onto the computational results are linear.

5.8. What characters do the solutions have? If $b \neq 0.5$ the function

$$
\omega_{1}^{2} / \check{\omega}_{1}^{2}\left(\mathcal{N} / \mathcal{N}_{\text {crit }}\right)
$$

is non-linear, but the deviation from the linearity is not significant at all. It becomes smaller when $b \longrightarrow 0.5$. If $b=0.5$ the half beam behaves as if it were a simply supported beam for which the function $\omega_{1}^{2} / \breve{\omega}_{1}^{2}\left(\mathcal{N} / \mathcal{N}_{\text {crit }}\right)$ is, in principal, a linear one. This fact is reflected in equations (5.9).

\section{Concluding Remarks}

The present paper clarifies what effect the axial load (compressive or tensile) has on the eigenfrequencies of a heterogeneous pinned-pinned beam with an intermediate roller support. This problem is, in fact, a three-point boundary value problem (eigenvalue problem) associated with homogeneous boundary conditions. After determining the Green function of the three-point boundary value problem the eigenvalue problem that provides the eigenfrequencies for the PrsP beam is transformed into an eigenvalue problem governed by a homogeneous Fredholm integral equation with the Green 
function as its kernel. The later eigenvalue problem is reduced to an algebraic eigenvalue problem which we solved numerically by using an effective solution algorithm based on the boundary element method. We have derived polynomial approximations for the sought function $\omega_{1}^{2} / \check{\omega}_{1}^{2}\left(\mathcal{N} / \mathcal{N}_{\text {crit }}\right)$.

\section{REFERENCES}

1. I. Ecsedi and D. Gönczi. "Thermoelastic stresses in nonhomogeneous prismatic bars." Annals of Faculty of Engineering Hunedoara - International Journal of Engineering, 13(2), (2015), pp. 49-52.

2. I. Ecsedi and A. Baksa. "Deformation of a cantilever curved beam with variable cross section." Journal of Computational and Applied Mechanics, 16(1), (2021), pp. 23-36. URL: 10.32973/jcam.2021.006.

3. H. K. Kim and M. S. Kim. "Vibration of beams with generally restrained boundary conditions using Fourier series." Journal of Sound and Vibration, 245(5), (2001), pp. 771-784. DOI: 10.1006/jsvi.2001.3615.

4. A. Bizzi, E. L. Fortaleza, and T. S. N. Guenka. "Dynamics of heavy beams: Closed-form vibrations of gravity-loaded Rayleigh-Timoshenko columns." Journal of Sound and Vibration, 510, (2021), p. 116259. DOI: 10.1016/j.jsv.2021. 116259 .

5. V. Ondra and B. Titurus. "Free vibration and stability analysis of a cantilever beam axially loaded by an intermittently attached tendon." Mechanical Systems and Signal Processing, 158, (2021), p. 107739. DOI: 10.1016/j.ymssp. 2021. 107739.

6. B. Bozyigit, Y. Yesilce, and S. Catal. "Free vibrations of axial-loaded beams resting on viscoelastic foundation using Adomian decomposition method and differential transformation." Engineering Science and Technology, an International Journal, 21(6), (2018), pp. 1181-1193. ISSN: 2215-0986. DOI: $10.1016 / \mathrm{j}$. jestch.2018.09.008.

7. A. Mirzabeigy and R. Madoliat. "Large amplitude free vibration of axially loaded beams resting on variable elastic foundation." Alexandria Engineering Journal, 55(2), (2016), pp. 1107-1114. DOI: 10.1016/j.aej.2016.03.021. URL: https: //www.sciencedirect.com/science/article/pii/S1110016816300345.

8. Jong-Shyong Wu and Bo-Hau Chang. "Free vibration of axial-loaded multi-step Timoshenko beam carrying arbitrary concentrated elements using continuousmass transfer matrix method." European Journal of Mechanics - A/Solids, 38, (2013), pp. 20-37. DOI: 10.1016/j.euromechsol.2012.08.003.

9. G. G. G. Lueschen, L. A. Bergman, and D. M. McFarland. "Green's functions for uniform Timoshenko beams." Journal of Sound and Vibration, 194(1), (1996), pp. 93-102. DOI: $10.1006 /$ jsvi.1996.0346.

10. M. A. Foda and Z. Abduljabbar. "A dynamic Green function formulation for the response of a beam structure to a moving mass." Journal of Sound and Vibration, 210(3), (1998), pp. 295-306. DOI: 10.1006/jsvi.1997.1334. 
11. S. Kukla and I. Zamojska. "Frequency analysis of axially loaded stepped beams by Green's function method." Journal of Sound and Vibration, 300(3), (2007), pp. 1034-1041. DOI: $10.1016 / \mathrm{j} \cdot \mathrm{jsv} .2006 .07 .047$.

12. G. Failla and A. Santini. "On Euler-Bernoulli discontinuous beam solutions via uniform-beam Green's functions." International Journal of Solids and Structures, 44(22), (2007), pp. 7666-7687. DOI: 10.1016/j.ijsolstr.2007.05.003.

13. A. Baksa and I. Ecsedi. "A note on the pure bending of nonhomogeneous prismatic bars." International Journal of Mechanical Engineering Education, 37(2), (2009), pp. 118-129. URL: 10.7227/IJMEE.37.2.4.

14. L. P. Kiss. "Vibrations and Stability of Heterogeneous Curved Beams." Ph.D Thesis. Institute of Applied Mechanics, University of Miskolc, Hungary, 2015. URL: http://geik.uni-miskolc.hu/intezetek/SALYI/ertekezesek.

15. G. Szeidl and L. Kiss. "Green Functions for Three Point Boundary Value Problems with Applications to Beams." Advances in Mathematics Research. Ed. by Albert R. Raswell. New York: Nova Science Publisher, Inc., 2020. Chap. Chapter 5, pp. 121-161.

16. J. M. Hoene-Wroński. Réfutation de la théorie des fonctions analytiques de Lagrange. Blankenstein, Paris, 1812.

17. L. Kiss, G. Szeidl, and M. Abderrazek. "Stability of heterogeneous beams with three supports - a novel approach to some classical stability problems." Unpublished manuscript. 2021.

18. G. Szeidl and L. P. Kiss. Mechanical Vibrations, an Introduction. Ed. by Vladimir I. Babitsky and Jens Wittenburg. Foundation of Engineering Mechanics. Springer Nature, Switzerland, 2005. Chap. 10. DOI: 10.1007/978-3-030-45074-8. 